Homology, Homotopy and Applications, vol.12(2), 2010, pp.109-146

\title{
ROUQUIER COMPLEXES ARE FUNCTORIAL OVER BRAID COBORDISMS
}

\author{
BEN ELIAS AND DAN KRASNER \\ (communicated by Michael A. Mandell)
}

Abstract

Using the diagrammatic calculus for Soergel bimodules developed by B. Elias and M. Khovanov, we show that Rouquier complexes are functorial over braid cobordisms. We explicitly describe the chain maps which correspond to movie move generators.

\section{Contents}

1 Introduction $\quad 109$

2 Constructions $\quad \mathbf{1 1 4}$

2.1 The Soergel Categorification . . . . . . . . . . . . . . . 114

2.2 Soergel Diagrammatics . . . . . . . . . . . . . . . . 115

2.3 Braids and Movies ..................... 120

2.4 Rouquier Complexes ...................... 121

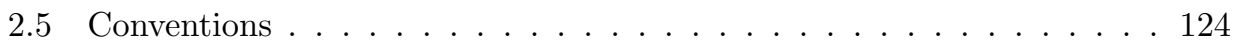

3 Definition of the Functor $\quad 125$

4 Checking the Movie Moves $\quad 130$

4.1 Simplifications ...................... 130

4.2 Movie Moves . . . . . . . . . . . . . . . . . . . . . 132

5 Additional Comments 143

5.1 The Benefits of Brute Force . . . . . . . . . . . . . . . . 143

5.2 Working over $\mathbb{Z}$.................... 145

\section{Introduction}

Let $\mathfrak{g}$ be a semisimple lie algebra, $W$ its Weyl group, $V$ the geometric representation of $W$ (i.e. the span of the root system), and $R$ the ring of polynomial functions on $V$, equipped with an action of $W$. Soergel in [14] introduced a full monoidal

Received July 29, 2009, revised February 6, 2010; published on September 16, 2010. 2000 Mathematics Subject Classification: 57M27, 20F36.

Key words and phrases: Soergel bimodule, Rouquier complex, link homology, braid cobordism.

This article is available at http://intlpress.com/HHA/v12/n2/a4

Copyright (c) 2010, International Press. Permission to copy for private use granted. 
subcategory $\mathcal{S C}$ of graded $R$-bimodules which was to play a significant role in the representation theory of $\mathfrak{g}$ and other related fields. There is an isomorphism between the (additive) Grothendieck ring of $\mathcal{S C}$ and the Hecke algebra $\mathcal{H}$ associated to $W$, where the Kazhdan-Lusztig generators $b_{i}$ of $\mathcal{H}$ lift to bimodules $B_{i}$ which are easily described. The full subcategory generated monoidally by these bimodules $B_{i}$ is here called $\mathcal{S C}_{1}$, and its objects are commonly referred to as Bott-Samelson bimodules. The category including grading shifts and direct sums of Bott-Samelson bimodules is refered to as $\mathcal{S C}_{2}$, and its idempotent closure, which contains all direct summands, is $\mathcal{S C}$; its objects are called Soergel bimodules.

Soergel bimodules are intrinsically linked to the geometry of flag varieties, hence their importance for geometric representation theory. See $[\mathbf{1 5}, \mathbf{1 6}, \mathbf{1 7}]$ for more details. We will focus on the strong connections between $\mathcal{S C}_{2}$ and knot theory that have come to light more recently, and trace the history below.

Let $\mathrm{Fr}$ denote the free monoid generated by overcrossings and undercrossings on $n+1$ strands. Let $B r$ denote the braid group, the quotient of $F r$ by the braid relations. We distinguish between a braid, which is an element of $\mathrm{Br}$, and a braid diagram, which is an element of $F r$, or a word in crossings. Let $\mathbb{1}$ denote the identity of $F$.

An action of a group $G$ on a category $\mathcal{C}$ is, for every $g \in G$, an endofunctor $\mathcal{F}_{g}$ of $\mathcal{C}$ such that $\mathcal{F}_{g} \circ \mathcal{F}_{h}$ is isomorphic to $\mathcal{F}_{g \circ h}$. The philosophy of categorification tells us that one can often enrich the structure by understanding the natural transformations between functors, like the relations between these isomorphisms. In the case of $\mathrm{Br}$, the category of braid cobordisms BrCob gives us a reasonable notion of what this extra structure should be. This category has braid diagrams as objects (viewed as 1-manifolds with boundary), and cobordisms of these diagrams as morphisms. The relations of the braid group lift to isomorphisms in BrCob. Therefore, we may define a functorial braid group action as a functor from $\mathbf{B r C o b}$ to the category of endofunctors of $\mathcal{C}$ (morphisms are natural transformations); on isomorphism classes, this returns an action of $\mathrm{Br}$ on $\mathcal{C}$. The introduction to [8] contains a nice general description of this concept.

The category $\mathbf{B r C o b}$ is equivalent to a combinatorially defined category, where cobordisms are replaced by movies (see Carter-Saito, [2]). For instance, the Reidemeister 3 move on a braid diagram is now a movie between two objects, whose inverse is another Reidemeister 3 move. To make a braid group action functorial would be to construct, for each movie between diagrams $g$ and $h$, a natural transformation between $\mathcal{F}_{g}$ and $\mathcal{F}_{h}$, such that these natural transformations satisfy the same relations as movies do (relations amongst movies are typically called movie moves).

Rouquier, in [13], discovered that one could use complexes of Soergel bimodules to construct an action of the braid group. To the $i^{\text {th }}$ overcrossing (resp. undercrossing) Rouquier associates a complex, which has $R$ in homological degree 0 and $B_{i}$ in homological degree -1 (resp. 1). To a word in these generators, one associates the tensor product of these basic complexes. For $w \in F r$ we denote by $\mathcal{F}_{w}$ the Rouquier complex for $w$, viewed as an object in the homotopy category of $\mathcal{S C}_{2}$. We also have a fully faithful functor from the homotopy category of $\mathcal{S C}_{2}$ to the endofunctor category of the homotopy category of $R$-bimodules, given by tensoring with a complex; we abuse notation and use $\mathcal{F}_{w}$ to refer to this functor as well. Rouquier showed that two 
words in $\mathrm{Fr}$ which are equal in $\mathrm{Br}$ correspond to homotopic complexes, and therefore we get an action of $B r$ on the homotopy category of $R$-bimodules.

Note that the complex $\mathcal{F}_{\mathbb{1}}$ is simply $R$ in homological degree 0 . Therefore the (degree 0 ) endomorphisms of $\mathcal{F}_{\mathbb{1}}$ are just multiplication by scalars. Thanks to certain adjunction properties, the endomorphisms of any functor $\mathcal{F}_{w}$ will consist only of rescaling, and the space of maps modulo homotopy between complexes associated to equivalent words in the braid group will be 1-dimensional.

On a seemingly unrelated note, M. Khovanov and L. Rozansky in [6] and [7] discovered a new invariant of tangles. To a tangle, one associates a matrix factorization, which is essentially a 2-periodic complex. In [6], the corresponding link homology theory is a categorification of the $s l(n)$-polynomial and in $[7]$ that of the HOMFLYPT polynomial. Moreover, they show (see Ch. 9 and 10 of [6]) that this theory is projectively functorial or functorial up to sign on tangle cobordisms for $s l(n)$-link homology and, similarly, on braid cobordisms for HOMFLY-PT link homology. That is, for every cobordism $S$ of tangles one can associate a map $\phi_{S}$ of matrix factorizations, and for a composition of cobordisms one has $\phi_{S_{1}} \circ \phi_{S_{2}}= \pm \phi_{S_{1} \circ S_{2}}$. The proof of this equality is simple and abstract. Using arguments similar to the previous paragraph, one shows that the space of matrix factorization maps is 1-dimensional, and thus these two sides must agree up to a scalar. Since both sides are typically isomorphisms and the calculation may be done over $\mathbb{Z}$, this scalar must be \pm 1 . The maps $\phi$ are complicated, and while in theory the paper might give enough information to define them explicitly (in Ch 8-10), it would take an incredibly diligent reader to do so. Moreover, for a certain saddle cobordism $S$, the map $\phi_{S}$ (which they call $\eta$ in Ch. 9 of [6]) can not be defined in a consistent way as to avoid the sign issue above; in other words, functoriality must fail on tangle cobordisms, and only functoriality up to a sign can hold.

In [5], Khovanov united this matrix factorization theory with Rouquier's work. To obtain a knot from a braid one may take its closure, wrapping the top boundary around to the bottom. Given a word in the braid group, one can cycle a crossing from the beginning of the word to the end and this will not change its closure: the closure of $x y$ is the same as the closure of $y x$. When replacing a braid with its Rouquier complex, Khovanov made the key observation that taking the braid closure should effectively correspond to equating the left and right actions of $R$, also known as taking Hochschild homology, for which $H H(M \otimes N) \cong H H(N \otimes M)$. Rouquier complexes naturally have two gradings, the homological grading and the internal grading of Soergel bimodules, and taking their Hochschild homology adds a third grading (its own homological grading). Khovanov showed that, up to degree shifts, this construction yields an equivalent triply-graded complex to the one produced by Khovanov-Rozansky HOMPLY-PT link homology for that braid closure.

Khovanov and Thomas in $[8]$ demonstrate numerous places where braid group actions on categories lift functorially or projectively functorially to braid cobordisms. Section 3.3 deals with the case of Rouquier complexes, where they show that Rouquier complexes lift projectively functorially. The proof is again the same (at least, when it comes to checking most movie moves): knowing that Hom spaces are 1-dimensional and that an isomorphism exists, any choice of isomorphism for the movie will satisfy the movie moves up to a sign. However, nothing is done explicitly: the only maps 
of complexes which are given are the simple ones corresponding to birth and death movies. To our knowledge, no one has bothered to take the functoriality maps of Khovanov and Rozansky and translate them to the context of Rouquier complexes, nor is it clear how this would be done. Khovanov and Thomas do suggest that many of these projective functors can be made into genuine functors, although their method of proof does not lend itself to showing this.

Recently, in [4], the first author in conjunction with Khovanov gave a presentation of the category $\mathcal{S C}_{1}$ in terms of generators and relations. It was shown that the entire category can be drawn graphically, thanks to the biadjointness and cyclicity properties that the category possesses. Each $B_{i}$ is assigned a color, and a tensor product is assigned a sequence of colors. Morphisms between tensor products can be drawn as certain colored graphs in the plane, whose boundaries on bottom and top are the sequence of colors associated to the source and target. Morphisms are invariant under isotopy of the graph embedding, and satisfy a number of other relations, described herein. In addition to providing a presentation, this graphical description is useful because one can use pictures to encapsulate a large amount of information; complicated calculations involving compositions of morphisms can be visualized intuitively and written down suffering only minor headaches.

Because of the simplicity of the diagrammatic calculus, we were able to calculate explicitly the chain maps which correspond to each generating cobordism in the braid cobordism category, and check that these chain maps satisfy the same relations that braid cobordisms do. The general proofs are straightforward and computationally explicit, performable by any reader with patience, time, and colored chalk. This makes the results of Rouquier and Khovanov that much more concrete, and implies the following new result.

Theorem 1.1. There is a (genuine) functor $\mathcal{F}$ from the category of combinatorial braid cobordisms to the category of complexes in $\mathcal{S C}_{2}$ up to homotopy, lifting Rouquier's complexes.

In fact, this functor is essentially unique, with the freedom being a choice of scalar and a choice of sign.

To save time and avoid extra calculations, we too will on occasion use the 1dimensionality of Hom spaces. Knowing that two maps are homotopic up to a scalar, we use the machinery developed by Clark, Morrison, and Walker [3], which allows one to calculate the scalar in question on a certain summand of a complex. The alternative would be to multiply eight $6 \times 6$ matrices by hand, several times. However, this machinery is unnecessary, and explicit calculations (and a LOT of colored chalk) could replace it.

This introduction has motivated Theorem 1.1 by discussing the links between Rouquier complexes and link homology. HOMFLY-PT link homology is an important link invariant. Many computations of HOMFLY-PT link homology were done by B. Webster [19], and by J. Rasmussen in [11] and [12]. In the latter paper [12], Rasmussen showed that given a braid presentation of a link, for every $n \in \mathbb{N}$ there exists a spectral sequence with $E^{1}$-term its HOMFLY-PT homology and the $E^{\infty}$-term its $s l(n)$ homology. This was a spectacular development in understanding the structural properties of these theories, and has also proven very useful in computation (see for 
example [10]). In order to apply our pictures to help calculate link homology, one should also come up with a diagrammatic picture for the higher Hochschild homology of Soergel bimodules. At the given moment this does not yet exist, although some insights have emerged. When the complete picture is developed, it should hopefully give an explicit and easily computable description of HOMFLY-PT link homology and functoriality therein, and possibly also the spectral sequences to other link homologies.

The careful reader might worry that functoriality of link homology would contradict the statement of [6], mentioned earlier, that their invariant of tangle cobordisms has an unavoidable sign issue. There are two ways of moving from braids to links: thinking of the braids in a larger category of tangles (which has cups and caps), or taking the braid closure. The former will lead to additional cobordisms dealing with cups and caps, like the saddle and its map $\eta$ mentioned above, which ruin functoriality. The latter consists, for Rouquier complexes, in applying the Hochschild homology functors. As functors, they will naturally preserve the extra structure of braid cobordism maps. We have shown that, for links which come from braid closures, their HOMFLY-PT link homology is functorial over cobordisms which come from braid cobordisms. This does not contradict $[\mathbf{6}]$.

Soergel bimodules are generally defined over certain fields $\mathbb{k}$ in the literature, because one is usually interested in Soergel bimodules as a categorification of the Hecke algebra, and in relating indecomposable bimodules to the Kazhdan-Lusztig canonical basis. However, we invite the reader to notice that the diagrammatic construction in [4] can be made over any ring, and in particular over $\mathbb{Z}$. In fact, all our proofs of functoriality still work over $\mathbb{Z}$. We discuss this in detail in section 5.2. In [9], the second author uses the work done here to define HOMFLY-PT and $s l(n)$-link homology theories over $\mathbb{Z}$, a construction which is long overdue. We also plan to investigate the Rasmussen spectral sequence in this context.

The organization of this paper is as follows. In Section 2 we go over all the previous constructions that are relevant to this paper. This includes the graphical presentation of $\mathcal{S C}$, the combinatorial braid cobordism category, and Rouquier's complexes which link $\mathcal{S C}$ to braids. In Section 2.5 we describe the conventions we will use in the remainder of the paper to draw Rouquier complexes for movies. In Section 3 we define the functor from the combinatorial braid cobordism category to the homotopy category of $\mathcal{S C}$, and in Section 4 we check the movie move relations to verify that our functor is well-defined. These checks are presented in numerical order, not in logical order, but a discussion of the logical dependency of the proofs, and of the simplifications that are used, can be found in Section 4.1. Section 5 contains some useful statements for the interested reader, but is not strictly necessary. Some additional light is shed on the generators and relations of $\mathcal{S C}$ in Section 5.1, where it is demonstrated how the relations arise naturally from movie moves. In Section 5.2 we briefly describe how one might construct the theory over $\mathbb{Z}$, so that future papers may use this result to define link homology theories over arbitrary rings. 


\section{Acknowledgements}

The authors were supported by NSF grants DMS-524460 and DMS-524124. We would like to thank the referee for comments on an earlier version of this paper, and M. Khovanov for his advice.

\section{Constructions}

\subsection{The Soergel Categorification}

For Soergel's original definition see [14], and for an easier version, see [17]. For more details, see $[\mathbf{4}]$.

Let $I$ be a finite subset of $\mathbb{Z}$. We work in type $A_{I}$, which is the sub-Dynkin diagram of $A_{\infty}$ given by vertices $I$. We say $i, j \in \mathbb{Z}$ are adjacent if $|i-j|=1$, and are distant if $|i-j| \geqslant 2$. We do not include $I$ in the notation when it is generic.

The Hecke algebra $\mathcal{H}(I)$ of type $A_{I}$ has a presentation as an algebra over $\mathbb{Z}\left[t, t^{-1}\right]$ with generators $b_{i}, i \in I$ and the Hecke relations

$$
\begin{aligned}
b_{i}^{2} & =\left(t+t^{-1}\right) b_{i} \\
b_{i} b_{j} & =b_{j} b_{i} \text { for distant } i, j \\
b_{i} b_{j} b_{i}+b_{j} & =b_{j} b_{i} b_{j}+b_{i} \text { for adjacent } i, j .
\end{aligned}
$$

Let $R(I)$ be the coordinate ring of the geometric representation of type $A_{I}$. Let us call the category of Bott-Samelson bimodules $\mathcal{S C}_{1}(I)$. It is a full monoidal subcategory of $R(I)$-bimodules, whose objects are all free as left $R(I)$-modules, which is generated by certain objects $B_{i}, i \in I$. These objects satisfy

$$
\begin{aligned}
& B_{i} \otimes B_{i} \cong B_{i}\{1\} \oplus B_{i}\{-1\} \\
& B_{i} \otimes B_{j} \cong B_{j} \otimes B_{i} \text { for distant } i, j \\
& B_{i} \otimes B_{j} \otimes B_{i} \oplus B_{j} \cong B_{j} \otimes B_{i} \otimes B_{j} \oplus B_{i} \text { for adjacent } i, j .
\end{aligned}
$$

The Grothendieck group of $\mathcal{S C}_{1}$ (or rather, of its additive and grading closure) is isomorphic to $\mathcal{H}$, with $\left[B_{i}\right]$ being sent to $b_{i}$, and $[R\{1\}]$ being sent to $t$.

We write the monomial $b_{i_{1}} b_{i_{2}} \cdots b_{i_{d}} \in \mathcal{H}$ as $b_{\boldsymbol{i}}$ where $\underline{\boldsymbol{i}}=i_{1} \ldots i_{d}$ is a finite sequence of indices; by abuse of notation, we sometimes refer to this monomial simply as $\underline{i}$. If $\underline{\boldsymbol{i}}$ is as above, we say the monomial has length $d$. We call a monomial non-repeating if $i_{k} \neq i_{l}$ for $k \neq l$. The empty set is a sequence of length 0 , and $b_{\emptyset}=1$. Similarly, write $B_{i_{1}} \otimes \cdots \otimes B_{i_{d}}$ as $B_{\boldsymbol{i}}$. Note that $B_{\emptyset}=R$, the monoidal identity.

Each object in $\mathcal{S C}_{1}$ has a biadjoint with respect to $\otimes$, and $B_{i}$ is self-biadjoint. Let $\omega$ be the $t$-antilinear anti-involution on $\mathcal{H}$ which fixes $b_{i}$, i.e. $\omega\left(t^{a} b_{\underline{i}}\right)=t^{-a} b_{\sigma(\underline{i})}$ where $\sigma$ reverses the order of a sequence. Then the contravariant functor sending an object to its biadjoint will descend on the Grothendieck group to $\omega$.

Let $\epsilon: \mathcal{H} \rightarrow \mathbb{Z}\left[t, t^{-1}\right]$ be the $\mathbb{Z}\left[t, t^{-1}\right]$-linear map which is uniquely specified by $\epsilon(x y)=\epsilon(y x)$ for all $x, y \in \mathcal{H}$ and $\epsilon\left(b_{\underline{i}}\right)=t^{d}$, whenever $\underline{i}$ is a non-repeating sequence of length $d$. Let $():, \mathcal{H} \times \mathcal{H} \rightarrow \mathbb{Z}\left[t, t^{-1}\right]$ be the map which sends $(x, y) \mapsto \epsilon(\omega(x) y)$. These structures (the pairing, $\omega, \epsilon$ ) clearly commute with various inclusions $\mathcal{H}(I) \subset$ $\mathcal{H}\left(I^{\prime}\right)$.

One useful feature of $\mathcal{S C}_{1}$ is that it is easy to calculate the dimension of Hom spaces in each degree. Let $\operatorname{HOM}(M, N) \stackrel{\text { def }}{=} \bigoplus_{m \in \mathbb{Z}} \operatorname{Hom}(M, N\{m\})$ be the graded 
vector space (actually an $R$-bimodule) generated by homogeneous morphisms of all degrees. Because each $B_{\underline{i}}$ is free as a left $R$-bimodule, $\operatorname{HOM}\left(B_{\underline{i}}, B_{\underline{j}}\right)$ is a free left $R$-module, and its graded rank over $R$ turns out to be $\left(b_{\underline{i}}, b_{\underline{j}}\right)$.

Remark 2.1. Soergel's proof of the compatibility of $($,$) and HOM is geometric. For [4],$ proving the equivalence of their diagrammatic category and Soergel's category was effectively proving that the diagrammatic category also was compatible with (, ) (the proof of which used a cognate of Theorem 2.7 below). However, once this compatibility is shown, calculating the size of HOM spaces is easy, and by counting dimensions one can show Theorem 2.7 and similar results. We will use this to show that certain Hom spaces have dimension 1 or 0 (in specific degrees), which will help to classify morphisms and homotopies between Rouquier complexes.

For two subsets $I \subset I^{\prime} \subset \mathbb{Z}$, the categories $\mathcal{S C}(I)$ and $\mathcal{S C}\left(I^{\prime}\right)$ are embedded in bimodule categories over different rings $R(I)$ and $R\left(I^{\prime}\right)$, but there is nonetheless a faithful inclusion of categories $\mathcal{S C}(I) \rightarrow \mathcal{S C}\left(I^{\prime}\right)$. This functor is not full: the size of $R$ itself will grow, and $\operatorname{HOM}\left(B_{\emptyset}, B_{\emptyset}\right)=R$. However, the graded rank over $R$ does not change, since the value of $\epsilon$ does not change over various inclusions. Effectively, the only difference in Hom spaces under this inclusion functor is base change on the left, from $R(I)$ to $R\left(I^{\prime}\right)$. We say the inclusion functor is fully faithful after base change.

As a result of this, most calculations involving morphisms between Soergel bimodules will not depend on which $I$ we work over. A graphical version of this property is forthcoming. In particular, the calculations we do for the braid group on $m$ strands will also work for the braid group on $m+1$ strands, and so forth.

\subsection{Soergel Diagrammatics}

We review the diagrammatic presentation of $\mathcal{S C}_{1}$ by generators and relations, found in [4]. See that paper for further details. We will not describe the equivalence between this presentation and that of Soergel. We will first deal with the case where $I=\{1,2, \ldots, n\}$, and then discuss what the inclusions of categories from the previous section imply for the general setting.

Remark 2.2. Technically, $\mathcal{S C}_{1}$ is equivalent to Soergel's original construction, but it is not the same as the category in [4], which we call $\mathcal{S C}_{1}^{\prime}$. The latter is a category of $R^{\prime}$ bimodules, where $R^{\prime}=\mathbb{k}\left[x_{1}, \ldots, x_{n+1}\right]$ is the coordinate ring of the standard representation of $S_{n+1}$, not the geometric representation. Our $\operatorname{ring}$ is $R=R^{\prime} /\left(e_{1}\right)$, where $e_{1}=x_{1}+\cdots+x_{n+1}$. Any symmetric polynomial is in the center of $\mathcal{S C}_{1}^{\prime}$, which means that taking the quotient of the category by $e_{1}$ is a simple affair. This is discussed briefly in [4], Section 4.5. So long as $n+1$ is invertible in $\mathbb{k}$, one can express the images of $x_{i}$ in $R$ as linear combinations of $f_{i} \in R$, where $f_{i}$ is the image of $x_{i}-x_{i+1}$ and corresponds precisely to the "double dot" colored $i$ in $\mathcal{S C}_{1}$ (see below). From this, it is fairly easy to define the equivalence of categories between $\mathcal{S C}_{1}$ and the quotient of $\mathcal{S C}_{1}^{\prime}$ by $e_{1}$. We leave it as an exercise to show that, when one rewrites the relations involving polynomials from $\mathcal{S C}_{1}^{\prime}$ in double dot form, one gets the dot sliding relations below.

When $n+1$ is not invertible (for instance, when working over $\mathbb{Z}$ ), $R$ is a proper $\mathbb{k}$-submodule of $R^{\prime} /\left(e_{1}\right)$. In this circumstance, working with $\mathcal{S C}_{1}$ (as defined below) 
is more natural than taking the $e_{1}$ quotient, and agrees with Soergel's original construction. See Section 5.2 for more about working over $\mathbb{Z}$.

An object in $\mathcal{S C}_{1}$ is given by a sequence of indices $\underline{\boldsymbol{i}}$, also called $B_{\underline{i}}$, which is visualized as $d$ points on the real line $\mathbb{R}$, labelled or "colored" by the indices in order from left to right. Morphisms are given by pictures embedded in the strip $\mathbb{R} \times[0,1]$ (modulo certain relations), constructed by gluing the following generators horizontally and vertically:

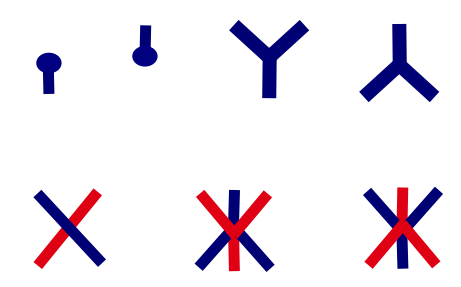

For instance, if "blue" corresponds to the index $i$ and "red" to $j$, then the lower right generator is a morphism from $j i j$ to $i j i$. The generating pictures above may exist in various colors, although there are some restrictions based on adjacency conditions.

With apologies to readers without access to a color version of this paper, we will refer to the darker color as "blue" and the lighter color as "red." Occassionally we will use a third color, which is represented with a dashed "orange" line.

We can view a morphism as an embedding of a planar graph, satisfying the following properties:

1. Edges of the graph are colored by indices from 1 to $n$.

2. Edges may run into the boundary $\mathbb{R} \times\{0,1\}$, yielding two sequences of colored points on $\mathbb{R}$, the top boundary $\underline{\boldsymbol{i}}$ and the bottom boundary $\boldsymbol{j}$. In this case, the graph is viewed as a morphism from $\underline{j}$ to $\underline{\boldsymbol{i}}$.

3. Only four types of vertices exist in this graph: univalent vertices or "dots", trivalent vertices with all three adjoining edges of the same color, 4-valent vertices whose adjoining edges alternate in colors between $i$ and $j$ distant, and 6 -valent vertices whose adjoining edges alternate between $i$ and $j$ adjacent.

The degree of a graph is +1 for each dot and -1 for each trivalent vertex. 4 -valent and 6 -valent vertices are of degree 0 . The term graph henceforth refers to such a graph embedding.

By convention, we color the edges with different colors, but do not specify which colors match up with which $i \in I$. This is legitimate, as only the various adjacency relations between colors are relevant for any relations or calculations. We will specify adjacency for all pictures, although one can generally deduce it from the fact that 6 -valent vertices only join adjacent colors, and 4 -valent vertices join only distant colors.

As usual in a diagrammatic category, composition of morphisms is given by vertical concatenation, and the monoidal structure is given by horizontal concatenation.

In writing the relations, it will be useful to introduce pictures for the "cup" and 
"cap":

$$
\begin{aligned}
& U=Y \\
& \bigcap=\ell
\end{aligned}
$$

We then allow $\mathbb{k}$-linear sums of graphs, and apply the relations below to obtain our category $\mathcal{S C}_{1}$. Some of these relations are redundant. For a more detailed discussion of the remarks in the remainder of this section, see [4].

$$
\begin{aligned}
& \eta=I=N \\
& \eta=\uparrow=\beta \\
& M=\lambda=M \\
& \omega=X=X \\
& (*)=*=\pi
\end{aligned}
$$

Remark 2.3. Any two embedded graphs which are connected by isotopy (fixing the boundary) are linked by the relations (8) through (12), so the morphism specified by a particular graph embedding only depends on its isotopy class. We could have described the category more simply by defining a morphism to be an isotopy class of a certain kind of planar graph. However, it is useful to understand that these "isotopy relations" exist, because they will appear naturally in the study of movie moves (see Section 5.1).

The embedded graphs constructed from our generating pictures can have no horizontal lines. However, we can allow embedded graphs with horizontal lines to represent any graph in its isotopy class which is constructible. Thanks to the isotopy relations, the resulting morphism is unambiguous.

$$
X=x
$$

Remark 2.4. Relation (13) effectively states that a certain morphism is invariant under 90 degree rotation. To simplify drawings later on, we often draw this morphism as follows:

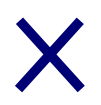

Note that morphisms will still be isotopy invariant with this convention. 
Here are the remainder of the one color relations.

$$
\begin{gathered}
Y=1=9 \\
\imath=0 \\
\imath+\mid \imath=2 !
\end{gathered}
$$

In the following relations, the two colors are distant.

$$
\begin{gathered}
X=\| \\
X=y \\
Y Y=Y \\
\imath=\mid \imath
\end{gathered}
$$

In this relation, two colors are adjacent, and both distant to the third color.

$$
\text { "NH" = "Pl" }
$$

In this relation, all three colors are mutually distant.

$$
\text { 义 }
$$

Remark 2.5. Relations (17) through (22) indicate that any part of the graph colored $i$ and any part of the graph colored $j$ "do not interact" for $i$ and $j$ distant. That is, one may visualize sliding the $j$-colored part past the $i$-colored part, and it will not change the morphism. We call this the distant sliding property.

In the following relations, the two colors are adjacent.

$$
\begin{aligned}
& y=b+b \\
& || \mid=\text { - }
\end{aligned}
$$




$$
\mathfrak{l}|-| \mathfrak{l}=-\frac{\mathfrak{l}}{\mathfrak{l}}+\mid \mathfrak{l}=\frac{1}{2}(|\mathfrak{l}-\mathfrak{l}|)
$$

The last equality in (26) is implied by (16), so it is not necessary to include as a relation. In this final relation, the colors have the same adjacency as $\{1,2,3\}$.

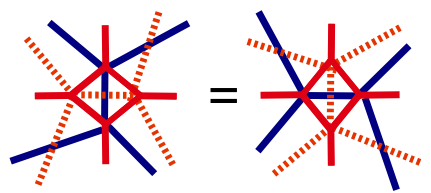

Remark 2.6. Because of isotopy invariance, the object $B_{i}$ in $\mathcal{S C}_{1}$ is self-biadjoint. In particular, instead of viewing the graph in $\mathbb{R} \times[0,1]$ as a morphism from $\underline{\boldsymbol{i}}$ to $\boldsymbol{j}$, we could twist it around and view it in the lower half plane (with no bottom boundary) as a morphism from $\emptyset$ to $\underline{\boldsymbol{i}} \sigma(\underline{\boldsymbol{j}})$.

We refer to any connected component of a graph which is a dot connected directly to the boundary as a boundary dot, and to any component equal to two dots connected by an edge as a double dot.

Relations (16), (20), and (26) are collectively called dot slides. They indicate how one might attempt to move a double dot from one region of the graph to another.

The following theorem and corollary are the hardest results from [4], and imply that HOM spaces are compatible with the pairing $($,$) on \mathcal{H}$.

Theorem 2.7. Morphisms where the color $i$ does not appear on the boundary are in the span of those graphs which do not include the color $i$ at all, except in double dots in the leftmost (alternatively, rightmost) region of the graph. This result may be obtained simultaneously for multiple indices $i$.

Corollary 2.8. The space $\operatorname{HOM}_{\mathcal{S C}_{1}}(\emptyset, \emptyset)$ is the free commutative polynomial ring generated by $f_{i}$, the double dot colored $i$, for various $i \in I$. This is a graded ring, where the degree of $f_{i}$ is 2 .

Remark 2.9. There is a natural identification of the polynomial ring of double dots and the coordinate ring $R$ of the geometric representation. Because of this, a combination of double dots is occasionally referred to as a polynomial. Placing double dots in the lefthand or righthand region of a diagram will correspond to the left and right action of $R$ on Hom spaces.

Remark 2.10. Consider $I \subset I^{\prime} \subset \mathbb{Z}$. If we have a morphism in $\mathcal{S C}_{1}\left(I^{\prime}\right)$ between two objects in $\mathcal{S C}_{1}(I)$, then each color $k \in I^{\prime} \backslash I$ does not appear in the boundary, so by Theorem 2.7 we can assume $k$ only appears in double dots on the left. The result is a morphism in $\mathcal{S C}_{1}(I)$, with extra colors appearing in double dots. This is the diagrammatic proof that $\operatorname{HOM}_{\mathcal{S C}(I)}(\underline{\boldsymbol{i}}, \underline{\boldsymbol{j}}) \otimes \mathbb{k}\left[f_{k}, k \in I^{\prime} \backslash I\right] \rightarrow \operatorname{HOM}_{\mathcal{S C}\left(I^{\prime}\right)}(\underline{\boldsymbol{i}}, \underline{\boldsymbol{j}})$ is an 
isomorphism, and the inclusion functor is fully faithful after base change. If we wished to define $\mathcal{S C}_{1}(I)$ for some $I \subset\{1, \ldots, n\}$, the correct definition would be to consider graphs which are only colored by indices in $I$.

We quickly note the diagrammatic proof for the isomorphisms (4) through (6). To show the equality

$$
\|=\frac{1}{2}(\dot{X}+\mathbf{X}) .
$$

use (14) to extend dots from the two lines toward each other, and then use (16) rotated by 90 degrees to connect the dots. This is a decomposition of $\mathrm{id}_{i i}$ into two orthogonal idempotents, each of which factor through $B_{i}$. This implies $B_{i} \otimes B_{i}=$ $B_{i}\{1\} \oplus B_{i}\{-1\}$. An alternative orthogonal decomposition is given by the following implication of (26), which involves an adjacent color.

$$
\|=(\dot{X}+\dot{X})-X
$$

Relation (17) expresses the isomorphism

$$
B_{i} \otimes B_{j}=B_{j} \otimes B_{i}
$$

for $i$ and $j$ distant.

Relation (24) is another orthogonal decomposition, and expresses the isomorphisms

$$
\begin{array}{r}
B_{i} \otimes B_{i+1} \otimes B_{i}=C_{i} \oplus B_{i} \\
B_{i+1} \otimes B_{i} \otimes B_{i+1}=C_{i} \oplus B_{i+1},
\end{array}
$$

where $C_{i}$ is an object in the Karoubi envelope $\mathcal{S C}$. We will not need the details, so we refer the reader to [4] for a better understanding.

We will primarily work within the category $\mathcal{S C}_{2}$, the additive graded version of $\mathcal{S C}_{1}$. However, since this includes fully faithfully into $\mathcal{S C}$, all calculations work there as well.

\subsection{Braids and Movies}

We will always use the combinatorial braid cobordism category BrCob as a replacement for the topological braid cobordism category. For details see Carter and Saito $[2]$.

The category of $(n+1)$-stranded braid cobordisms can be defined as follows. The objects are arbitrary sequences of braid group generators $O_{i}, 1 \leqslant i \leqslant n$, and their inverses $U_{i}=O_{i}^{-1}$. These sequences can be drawn using braid diagrams on the plane, where $O_{i}$ is an overcrossing (the $i+1^{\text {st }}$ strand crosses over the $i^{\text {th }}$ strand) and $U_{i}$ is an undercrossing. Objects have a monoidal structure given by concatenation of sequences. A movie is a finite sequence of transformations of two types:

I. Reidemeister type moves, such as

$$
\begin{gathered}
\tau_{1} O_{i} U_{i} \tau_{2} \leftrightarrow \tau_{1} \tau_{2}, \\
\tau_{1} O_{i} O_{j} \tau_{2} \leftrightarrow \tau_{1} O_{j} O_{i} \tau_{2} \text { for distant } i, j \\
\tau_{1} O_{i} O_{i+1} O_{i} \tau_{2} \leftrightarrow \tau_{1} O_{i+1} O_{i} O_{i+1} \tau_{2},
\end{gathered}
$$

where $\tau_{1}$ and $\tau_{2}$ are arbitrary braid words. 
II. Addition or removal of a single $O_{i}$ or $U_{i}$ from a braid word

$$
\tau_{1} \tau_{2} \leftrightarrow \tau_{1} O_{i}^{ \pm 1} \tau_{2}
$$

These transformations are known as movie generators. Morphisms in $\mathrm{BrCob}$ will consist of movies modulo two kinds of relations. The first are known as movie moves, and can be found in figures 1 and 2. Movie moves $1-10$ are composed of type I transformations and $11-14$ each contains a unique type II move. We denote the location of the addition or removal of a crossing in these last 4 movies by little black triangles. There are many variants of each of these movies: one can change the relative height of strands, can reflect the movie horizontally or vertically, or can run the movie in reverse. We refer the reader to Carter and Saito [2], section 3.

The second kind of relations are locality moves. Locality moves merely state that if two transformations are performed on a diagram in locations that do not interact (they do not share any of the same crossings) then one may change the order in which the transformations are performed. This merely insures that $\mathbf{B r C o b}$ is monoidal. Any potential functor from $\mathrm{BrCob}$ to a monoidal category $\mathcal{C}$ which preserves the monoidal structure will automatically satisfy the locality moves. Because of this, we need not mention the locality moves again.

Definition 2.11. Given a braid diagram $P$ (or an object in the cobordism category), the diagram $\bar{P}$ is given by reversing the sequence defining $P$, and replacing all overcrossings with undercrossings and vice versa. If one identifies $O_{i}$ as $U_{i}^{-1}$, then this corresponds precisely to taking the inverse in $\mathrm{Fr}$.

\subsection{Rouquier Complexes}

Rouquier defined a braid group action on the homotopy category of complexes in $\mathcal{S C}_{2}$ (see [13]). To the $i^{\text {th }}$ overcrossing, he associated a complex $B_{i}\{1\} \longrightarrow B_{\emptyset}$, and to the undercrossing, $B_{\emptyset} \longrightarrow B_{i}\{-1\}$. In each case, $B_{\emptyset}$ is in homological degree 0 . The differential in each complex is a boundary dot. Drawn graphically, these complexes look like:
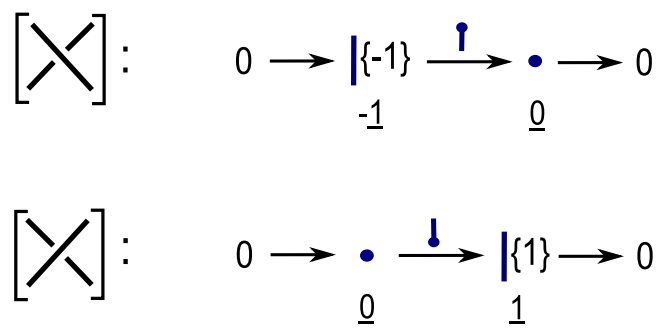

We are using some new and abusive notation: the (blue) circle here is a place holder for empty space, and the blue line indicates the object $B_{i}$ itself, not its identity morphism. See the next section for the conventions being used.

To a braid one associates the tensor product of the complexes for each crossing. Rouquier showed in [13] that the braid relations hold amongst these complexes. We will not assume this result.

The category that Rouquier complexes live in depends on the number of strands, but not in an essential way. Since adding strands is fully faithful after base change, 
BEN ELIAS AND DAN KRASNER
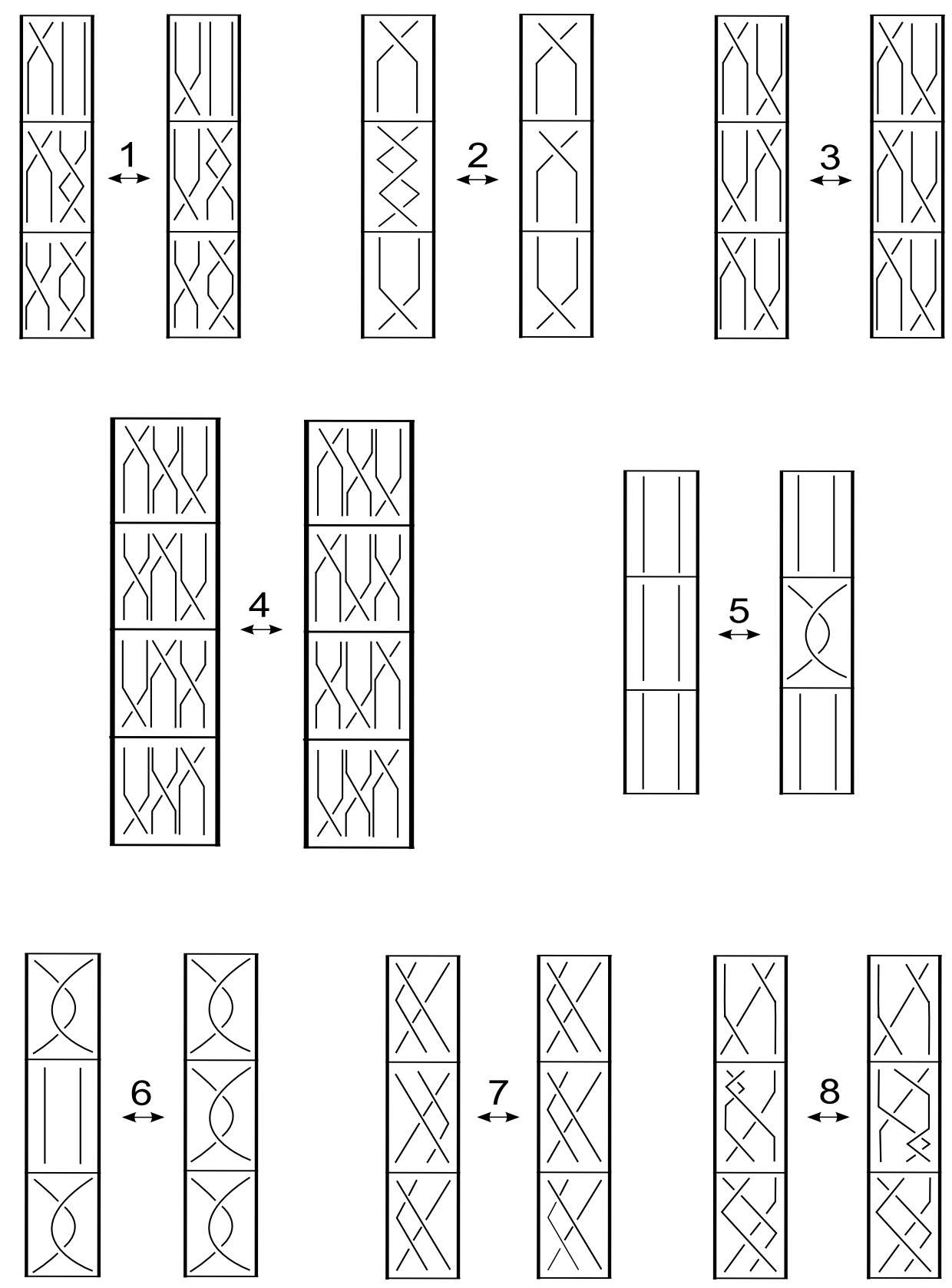

Figure 1: Braid movie moves $1-8$ 

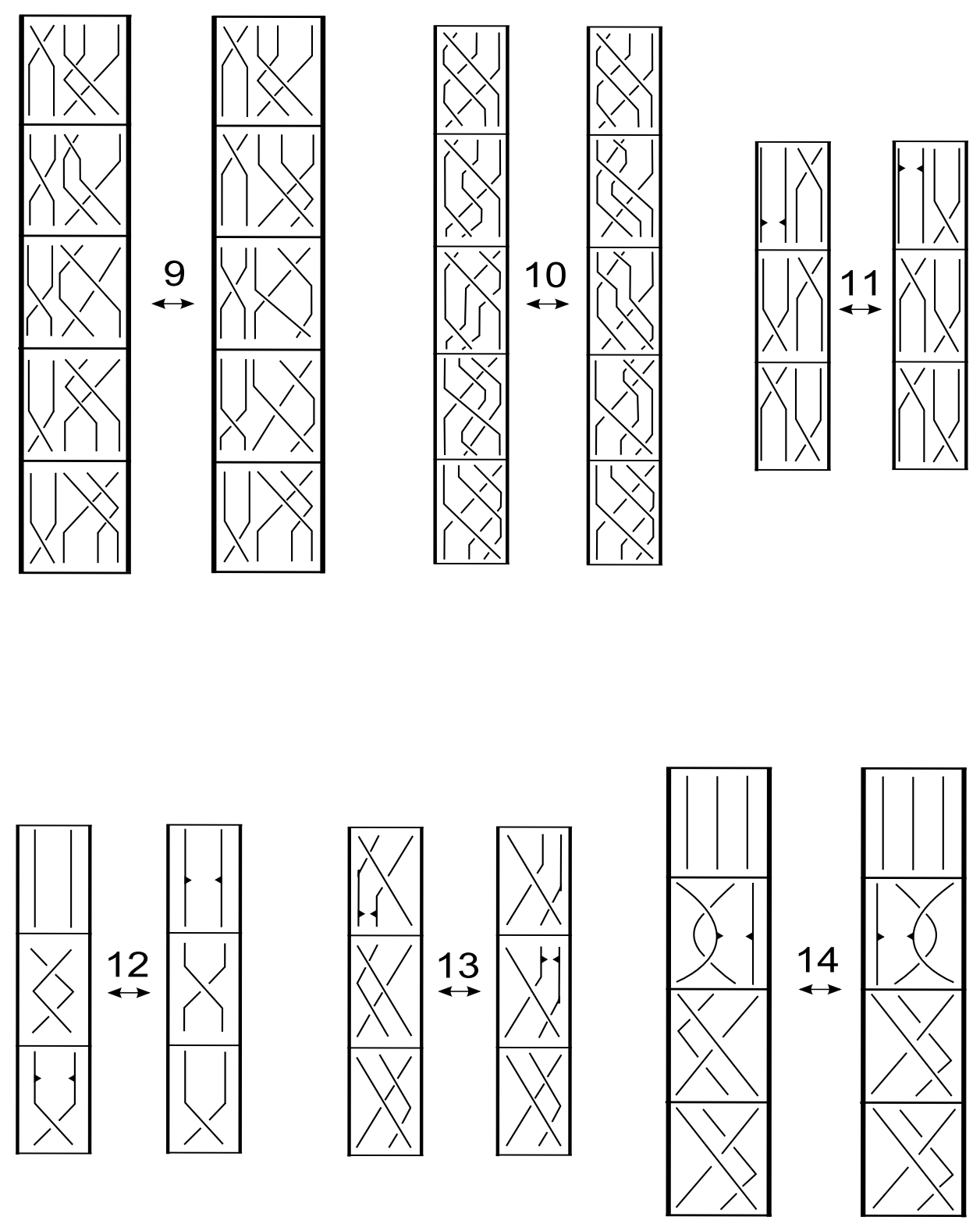

Figure 2: Braid movie moves $9-14$ 
it will only change the base ring but will not change the free rank of Hom spaces between two Rouquier complexes, or the pictures which describe specific morphisms. Therefore, all calculations involving Rouquier complexes are entirely "local" in that one can study only a part of a braid diagram.

\subsection{Conventions}

These are the conventions we use to draw Rouquier complexes henceforth.

An object in $\mathcal{S C}_{1}$ should be a sequence of colors (say, points on a line). Suppose we take the Rouquier complex for a braid $O_{i_{1}}^{ \pm} O_{i_{2}}^{ \pm} \ldots O_{i_{d}}^{ \pm}$. It is clear that summands in the complex correspond precisely to subsequences of $\underline{i}$, so that the object $B_{j}$ will appear exactly once for each subsequence $\boldsymbol{j}$ of $\underline{\boldsymbol{i}}$. These can be represented as points on a line, but it would be a headache to remember which subsequence corresponded to which object (after all, two distinct subsequences could yield the same object). Instead, we add colored placemarks which indicate that this particular $i_{k}$ was not in the subsequence.

Since it would be difficult to distinguish between colored points and colored placemarks, we cheat a little. We represent the object $B_{i}$ not as a point on a line, but by drawing its identity morphism $\mathbb{1}_{B_{i}}$, a vertical line. We represent a placemark with a colored circle, which for all practical purposes can be treated as the empty graph. This convention is used above in the definition of Rouquier complexes.

Each differential from one summand to another will be a dot map, with an appropriate sign coming from tensor product rules. This dot map either sends a line to a circle, or a circle to a line, using the convention above. We say the line/circle is switched.

1. The dot would be a map of degree 1 if $B_{i}$ had not been shifted accordingly. In $\mathcal{S C}_{2}$, all maps must be homogeneous, so we could have deduced the degree shift in $B_{i}$ from the degree of the differential. Because of this, it is not useful to keep track of various degree shifts of objects in a complex. We will draw all the objects without degree shifts, and all differentials will therefore be maps of graded degree 1 (as well as homological degree 1). It follows from this that homotopies will have degree -1 , in order to be degree 0 when the shifts are put back in. One could put in the degree shifts later, noting that $B_{\emptyset}$ always occurs as a summand in a tensor product exactly once, with degree shift 0 .

2. Similarly, one need not keep track of the homological dimension. $B_{\emptyset}$ will always occur in homological dimension 0 .

3. We will use blue for the index associated to the leftmost crossing in the braid, then red and dotted orange for other crossings, from left to right. The adjacency of these various colors is determined from the braid.

4. We read braid diagrams from bottom to top, and write tensor products from left to right. In figure 3, we take the complex for the blue crossing, and tensor by the complex for the red crossing. When translated into pictures, blue always appears to the left of red.

5. One can deduce the sign of a differential between two summands using the Liebnitz rule, $d(a b)=d(a) b+(-1)^{|a|} a d(b)$. In particular, since a line always occurs 

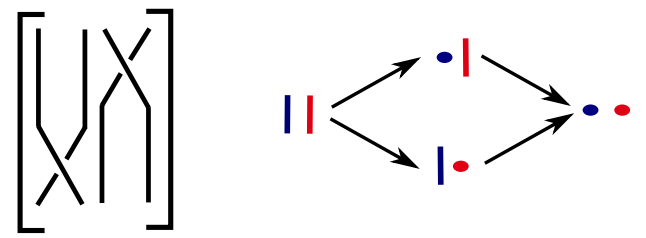

Figure 3: The blue complex tensor the red complex

in the basic complex in homological dimension \pm 1 , the sign on a particular differential is exactly given by the parity of lines appearing to the left of the map. For example,
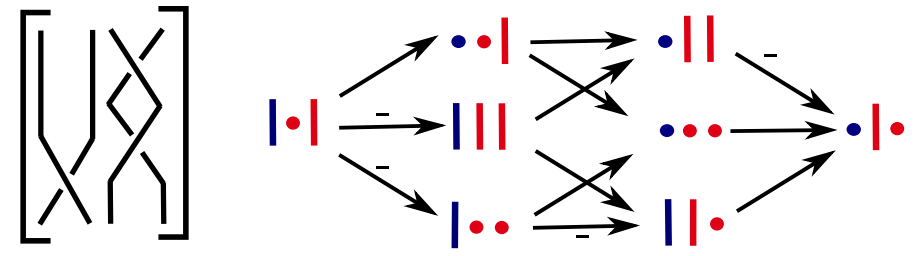

6. When putting an order on the summands in each homological degree, we use a lexicographic order. There is a unique summand of smallest homological degree $m$, which we take as our template. In homological degree $m+d$ there will be a total of $d$ switches, that is, lines turned to circles or circles to lines. Order the summands in that degree by which lines/circles were switched. For instance, when $d=2$, the first summand has the 1st and 2nd switches, the next has 1st and 3rd, and so forth.
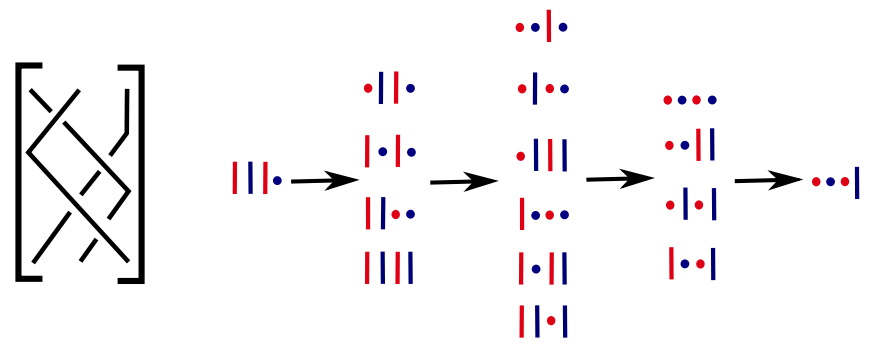

7. Starting in the next section and henceforth, a differential will always be a horizontal arrow, while a map of chain complexes will be a vertical arrow.

\section{Definition of the Functor}

We extend Rouquier's complexes to a functor $\mathcal{F}$ from the combinatorial braid cobordism category to the category of chain complexes in $\mathcal{S C}_{2}$ modulo homotopy. Rouquier already defined how the functor acts on objects, so it only remains to define chain maps for each of the movie generators, and check the movie move relations. 
There are four basic types of movie generators: birth/death of a crossing, slide, Reidemeister 2 and Reidemeister 3.

Birth and Death generators.

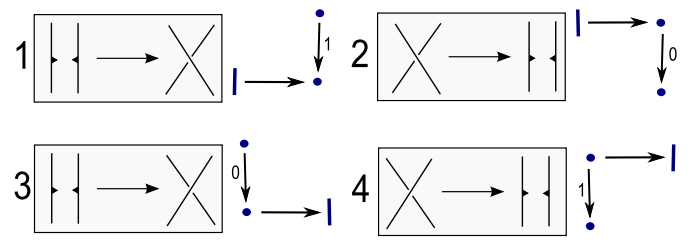

Reidemeister 2 generators.
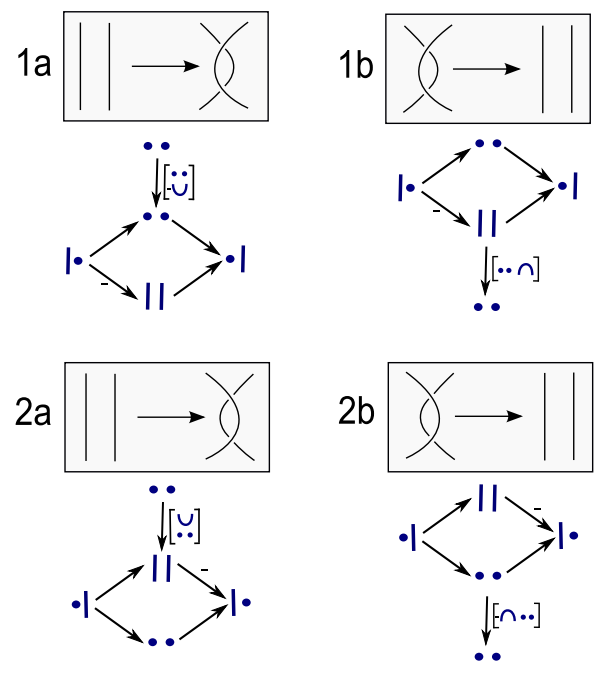

Slide generators.
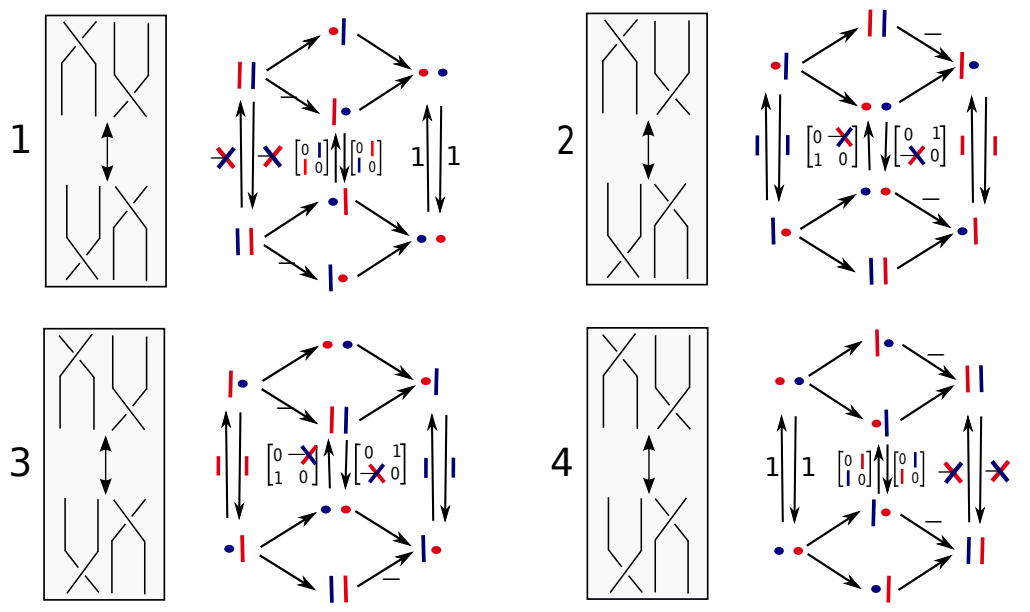
Reidemeister 3 generators. There are 12 generators in all: 6 possibilities for the height orders of the 3 strands (denoted by a number 1 through 6 ), and two directions for the movie (denoted "a" or "b"). Thankfully, the color-switching symmetries of the Soergel calculus allow us to explicitly list only 6 (see figures 4 and 5). The left-hand column lists the generators, and the chain complexes they correspond to; switching colors in the complexes yields the corresponding generator listed on the right. Each of these variants has a free parameter $x$, and the parameter used for each variant is actually independent from the other variants.

Remark 3.1. Using sequences of R2-type generators and various movie moves we could have abstained from ever defining certain R3-type variants or proving the movie moves that use them. We never use this fact, and list all on pages 128 and 129 for completeness.

Claim 3.2. Up to homotopy, each of the R3 generators is independent of $x$.

Proof. We prove the claim for generator 1a in figure 4; all the others follow from essentially the same computation. One can easily observe that there are very few summands of the source complex which admit degree -1 maps to summands of the target complex. In fact, the unique (up to scalar) non-zero map of homological degree -1 and graded degree -1 is a red trivalent vertex: a red fork which sends the single red line in the second row of the source complex to the double red line in the second row of the target complex. Given two chain maps, one with free variable $x$ and one with say $x^{\prime}$, the homotopy is given by the above fork map, with coefficient $\left(x-x^{\prime}\right)$. The homotopies for the other variants are exactly the same, save for the position, color, and direction of the fork (there is always a unique map of homological and graded degree -1).

Remark 3.3. In our personal work, we found by explicit calculation that the parameters $x$ are the only part of the whole ensemble which homotopy affects: the above maps (with choice of $x$ ) are the unique chain maps for which the movie move relations work, up to scalar. Abstract arguments will soon show that the choice is 1-dimensional modulo homotopy.

Remark 3.4. For all movie generators, there is a unique summand of both the source and the target which is $B_{\emptyset}$. We have clearly used the convention that for Type I movie generators, the induced map from the $B_{\emptyset}$ summand in the source to the $B_{\emptyset}$ summand in the target is the identity map. Ignoring this convention, each of the above maps may be multiplied by an invertible scalar. Some relations must be imposed between these scalars, which the reader can determine easily by looking at the movie moves (each side must be multiplied by the same scalar). Movie move 11 forces all slide generators to have scalar 1 . Movie move 13 forces all R3 generators to have scalar 1. Movie move 14 and 2 combined force the scalar for any R2 generator to be \pm 1 , and then movie moves 2 and 5 force this sign to be the same for all 4 variants. Movie move 12 shows that the scalar for the birth of an overcrossing and the death of an undercrossing are related by the sign for the $\mathrm{R} 2$ generator. So the remaining freedom in the definition of the functor is precisely a choice of one sign (R2) and one scalar (birth/death). 

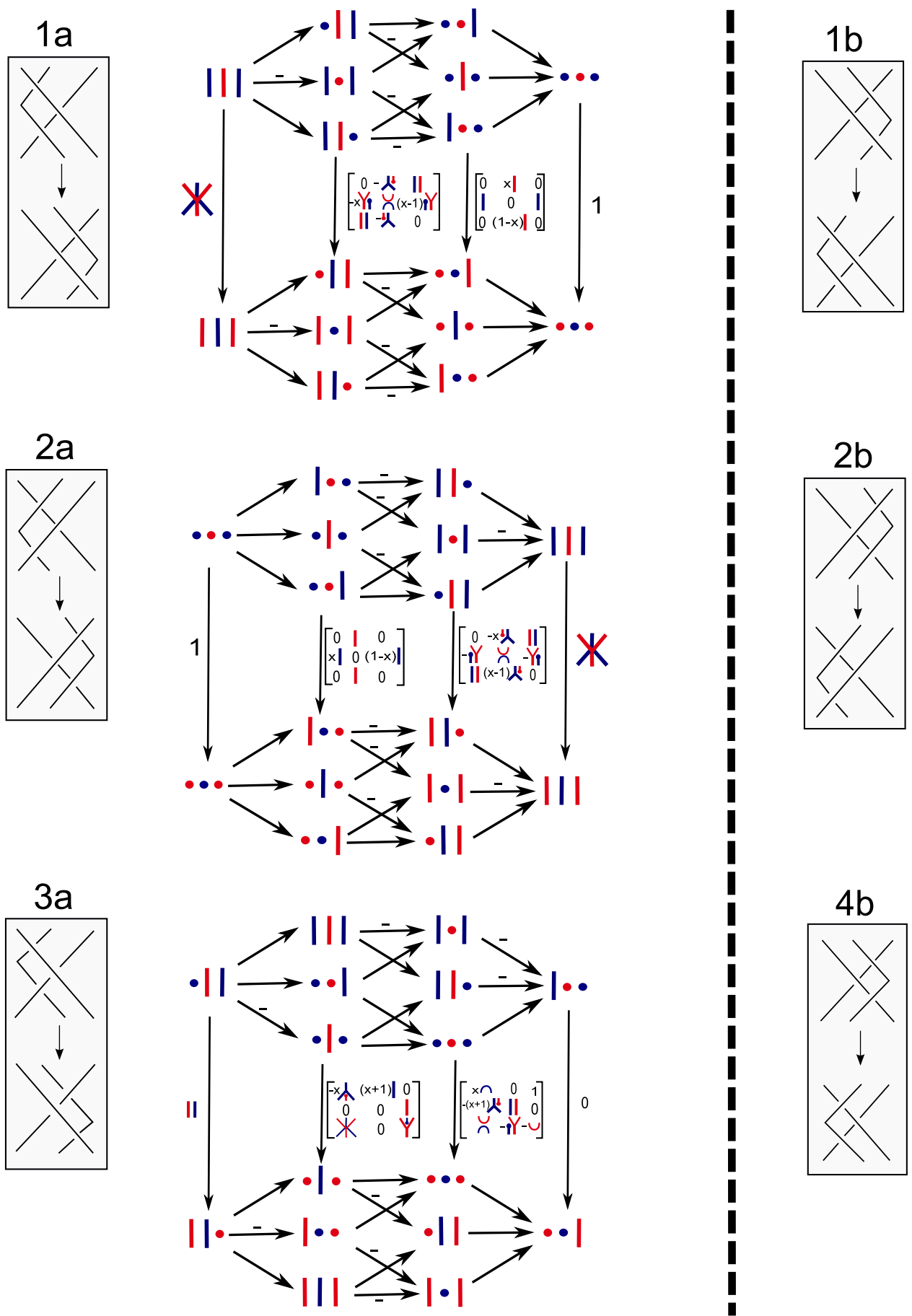

Figure 4: Reidemeister 3 type movie move generators 

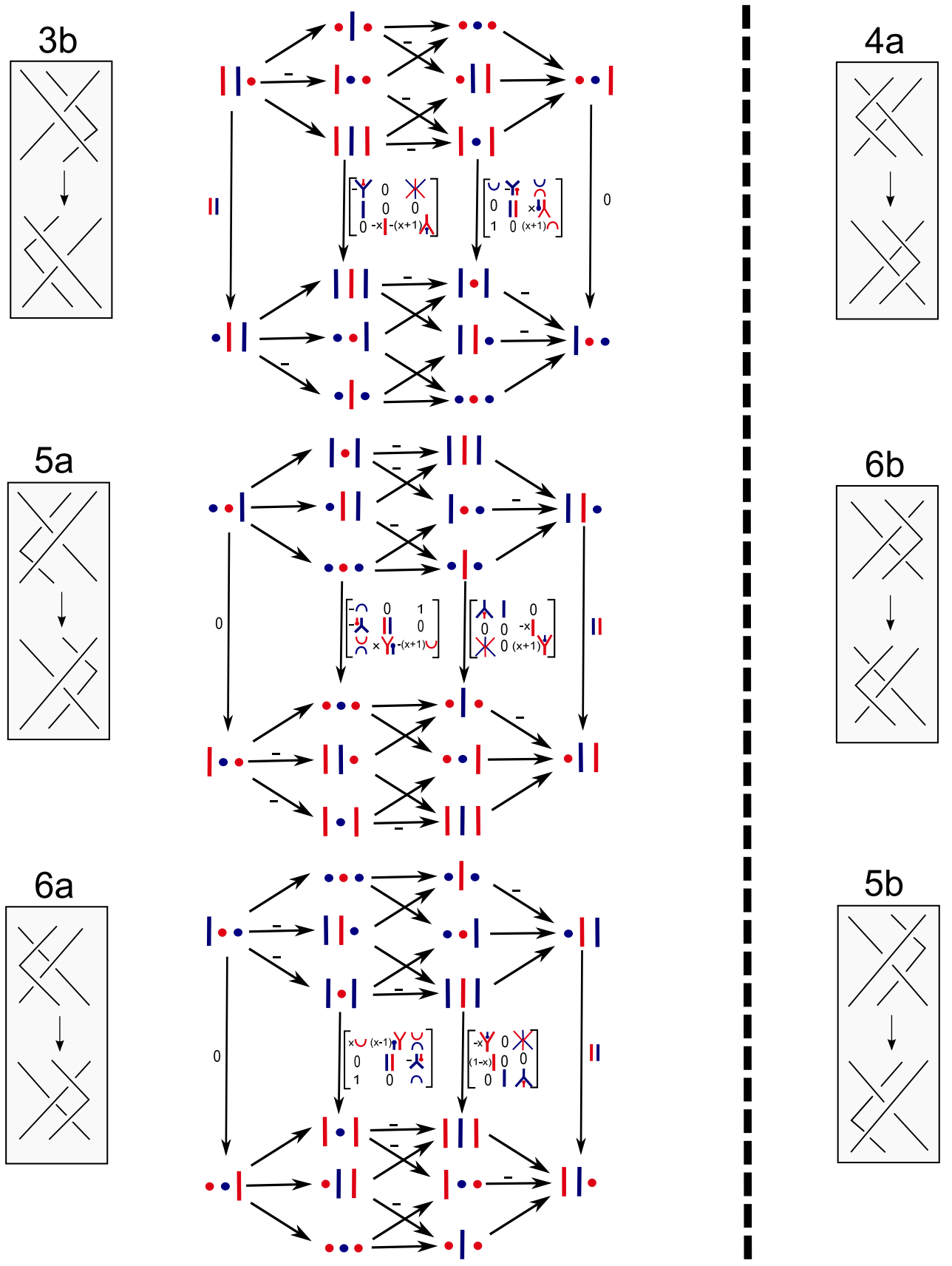

Figure 5: Reidemeister 3 type movie move generators 


\section{Checking the Movie Moves}

\subsection{Simplifications}

Since $\mathcal{F}$ has been defined explicitly, one can compose chain maps on both sides of a movie move and explicitly find the homotopy which gives the difference. In general, this is not difficult, though it is time-consuming. We do a number of calculations below, and have done even more in preparation for this paper (the interested reader is welcome to contact us for scans of our notes). Still, there are too many variants of too many movie moves to check them without resorting to a number of simplifications.

Thanks to Clark, Morrison, and Walker [3], a significant amount of work can be bypassed using a clever argument. We now repeat some results from that paper.

Notation 4.1. Let $P, Q, T$ designate braid diagrams. $\operatorname{Hom}(P, Q)$ will designate the hom space between $\mathcal{F}_{P}, \mathcal{F}_{Q}$ in the homotopy category of complexes in $\mathcal{S C}_{2}$. We write HOM for the graded vector space of all morphisms of complexes (not necessarily in degree 0$)$.

Lemma 4.2. (see [3]) Suppose that Movie Move 2 holds. Then there is an adjunction isomorphism $\operatorname{Hom}\left(P O_{i}, Q\right) \rightarrow \operatorname{Hom}\left(P, Q U_{i}\right)$, or more generally $\operatorname{Hom}(P T, Q) \rightarrow$ $\operatorname{Hom}(P, Q \bar{T})$. Similarly for other variations: $\operatorname{Hom}\left(O_{i} P, Q\right) \rightarrow \operatorname{Hom}\left(P, U_{i} Q\right)$, $\operatorname{Hom}\left(P, Q O_{i}\right) \rightarrow \operatorname{Hom}\left(P U_{i}, Q\right)$, etc.

Proof. Given a map $f \in \operatorname{Hom}\left(P O_{i}, Q\right)$, we get a map in $\operatorname{Hom}\left(P, Q U_{i}\right)$ as follows: take the R2 movie from $P$ to $P O_{i} U_{i}$, then apply $f \otimes \operatorname{id}_{U_{i}}$ to $Q U_{i}$. The reverse adjunction map is similar, and the proof that these compose to the identity is exactly Movie Move 2.

Corollary 4.3. For any braid diagram $P, \operatorname{Hom}(P, P) \cong \operatorname{Hom}(\mathbb{1}, P \bar{P})$.

Note that in the braid group, $P \bar{P}=\mathbb{1}$.

Lemma 4.4. Suppose that Movie Moves 3, 5, 6, and 7 hold. Then if $P$ and $Q$ are two braid diagrams which are equal in the braid group, then $\operatorname{Hom}(P, T) \cong \operatorname{Hom}(Q, T)$.

Proof. Movie Moves 3,5,6, and 7 together imply that if two braid diagrams are equal in the braid group, then they have isomorphic complexes.

The complex associated to $\mathbb{1}$ is just $B_{\emptyset}$ in homological degree 0 . So $\operatorname{HOM}(\mathbb{1}, \mathbb{1})=$ $\operatorname{HOM}\left(B_{\emptyset}, B_{\emptyset}\right)$, which we have already calculated is the free polynomial ring generated by double dots. In particular, the degree 0 morphisms are just multiples of the identity. We have now proven:

Corollary 4.5. Suppose that Movie Moves 2,3,5,6,7 all hold. If $P$ and $Q$ are braid diagrams which are equal in the braid group, then $\operatorname{Hom}(P, Q) \cong \mathbb{k}$, a one-dimensional vector space.

The practical use of finding one-dimensional Hom spaces is to apply the following method.

Definition 4.6. (See [3]) Consider two complexes $A$ and $B$ in an additive $\mathbb{k}$-linear category. We say that a summand of a term in $A$ is homotopically isolated with respect to $B$ if, for every possible homotopy $h$ from $A$ to $B$, the map $d h+h d: A \rightarrow B$ is zero when restricted to that summand. 
Lemma 4.7. Let $\phi$ and $\psi$ be two chain maps from $A$ to $B$, such that $\phi \equiv c \psi$ up to homotopy, for some scalar $c \in \mathbb{k}$. Let $X$ be a homotopically isolated summand of $A$. Then the scalar $c$ is determined on $X$, that is, $\phi=c \psi$ on $X$.

The proof is trivial, see [3]. The final result of this argument is the following corollary.

Corollary 4.8. Suppose that Movie Moves 2,3,5,6,7 all hold. If $P$ and $Q$ are braid diagrams which are equal in the braid group, and $\phi$ and $\psi$ are two chain maps in $\operatorname{Hom}(P, Q)$ which agree on a homotopically isolated summand of $P$, then $\phi$ and $\psi$ are homotopic.

Proof. Because the Hom space modulo homotopy is one-dimensional, we know there exists a constant $c$ such that $\phi \equiv c \psi$. The agreement on the isolated summand implies that $c=1$.

Type I movie generators are isomorphisms of complexes, and movie moves 1 through 10 therefore all consist of morphisms $P$ to $Q$, for $P$ and $Q$ equal in the braid group. Finding a homotopically isolated summand and checking the equality of the chain maps on that summand alone will greatly reduce any work that needs to be done. This can be applied to movie moves 1,4,8,9,10, since the others are assumed already. However, some calculations are easy enough to perform by brute force that we do them explicitly anyway.

One final simplification, also found in Clark, Morrison, and Walker, is that modulo Movie Move 8 all variants of Movie Move 10 are equivalent. Hence we can prove Movie Move 10 by investigating solely the overcrossing-only variant.

The simplifications above apply to any functorial theory of braid cobordisms, so long as $\operatorname{Hom}(\mathbb{1}, \mathbb{1})$ is one-dimensional. Now we look at what we can say specifically about homotopically isolated summands for Rouquier complexes.

Any homotopy must be a map of degree -1 (if we ignore degree shifts on objects, as in our conventions), which means that the corresponding graph must have a trivalent vertex. There are very few maps of negative degree in $\mathcal{S C}_{2}$, a fact which immediately forces most homotopies to be zero. The possible negative degree maps can be calculated using the pairing on $\mathcal{H}$, as discussed in Section 2.1. In fact, the only nonzero maps which occur in homotopies outside of Movie Move 10 are:

\section{$Y X Y X+Y$}

Here is an example of the usefulness of such methods:

Remark 4.9 (Proof of Movie Move 10). There are no negative degree maps from $B_{\emptyset}$ to $B_{i}$, for any $i$. In an overcrossing-only braid, $B_{\emptyset}$ occurs alone in the maximal homological grading, so that $d=0$ on that summand. The penultimate homological grading is a direct sum of various $B_{i}$, so that $h=0$ on $B_{\emptyset}$, and the summand is homotopically isolated. Moreover, the Reidemeister 3 chain map for overcrossings sends $B_{\emptyset}$ to itself by the identity map. Therefore, both sides of the overcrossing-only 
variant of Movie Move 10 act by the identity on this summand, and therefore are equal as chain maps. The remainder of the Movie Move 10 variants fall from Movie Move 8 .

\subsection{Movie Moves}

When discussing the possible variants of each movie move, we will ignore variants accomplished by a horizontal flip. These just correspond to color-changing symmetries, and do not affect any calculations. All variants involve changing the height order of the strands, performing a vertical flip, or doing the movie in reverse order. On occasion the vertical flip and the horizontal flip agree.

Note (Logical sequence in the proofs of the movie moves). We list the movie moves in numerical order, as opposed to logical order of interdependence. To use the technical lemma about homotopically isolated summands we first need to check movie moves $2,3,5,6,7$. The reader will see that we prove these through direct computation, relying on none of the other moves.

MM1. There are eight variants, of which we present two explicitly here. For the first (figure 6) we write out the matrices in full, and let the reader multiply them; for the second (figure 7) we just give the composition. In any variant, the slide generator behaves the same: a crossing will have coefficient -1 , an identity map coefficient 1 . Reversing direction uniformly changes the sign on the cups (or caps) in the R2 move. The only interesting part of the check uses a twist of relation (11). Note that every summand is homotopically isolated, so this check can be done on $B_{\emptyset}$, and is trivial.

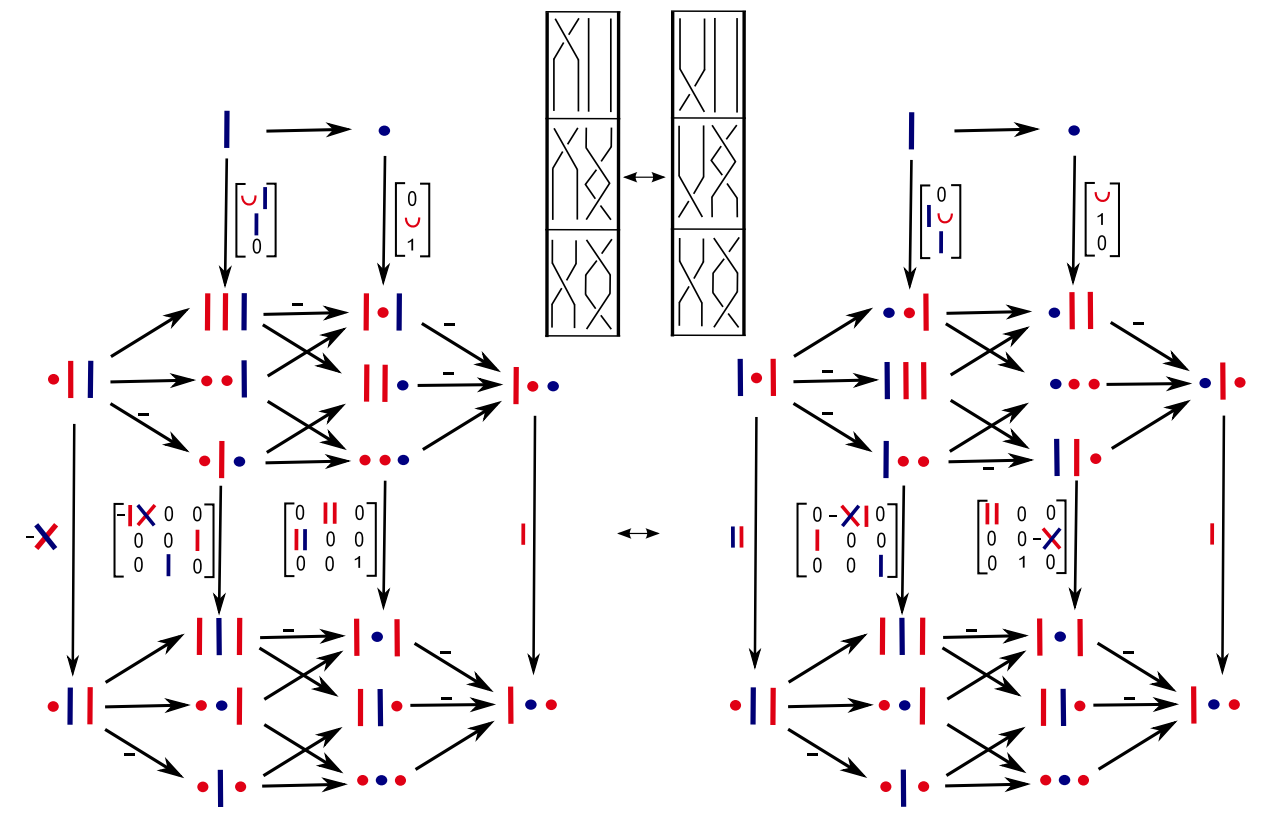

Figure 6: Movie Move 1 associated to slide generator 1 


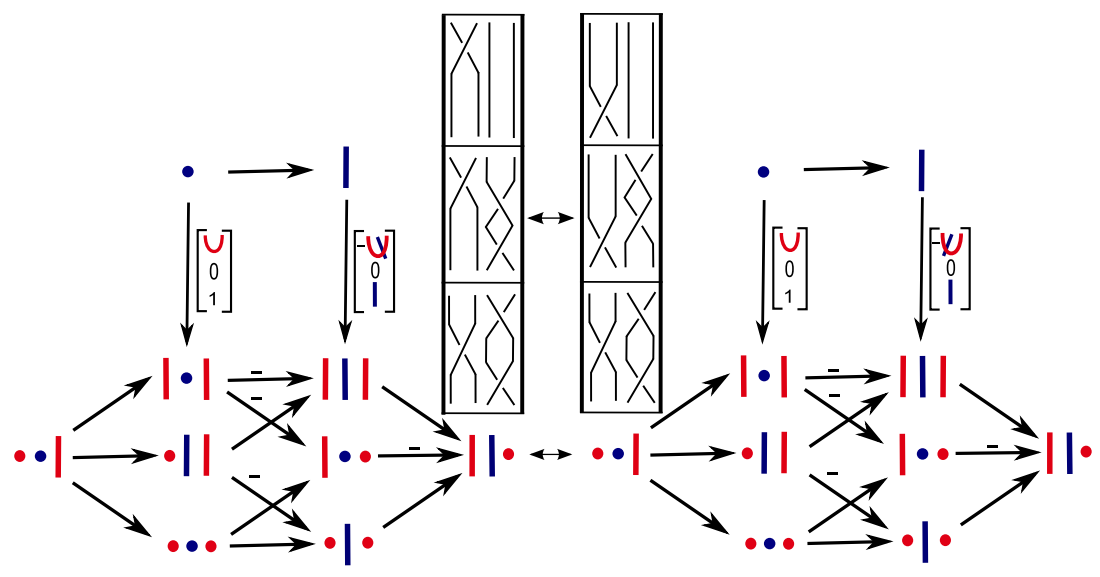

Figure 7: Movie Move 1 associated to slide generator 3

MM2. There are 4 variants to deal with here; we describe only one, and similar reasoning to that of MM1 will convince the reader that the other 3 are readily verified. The composition has the following form:

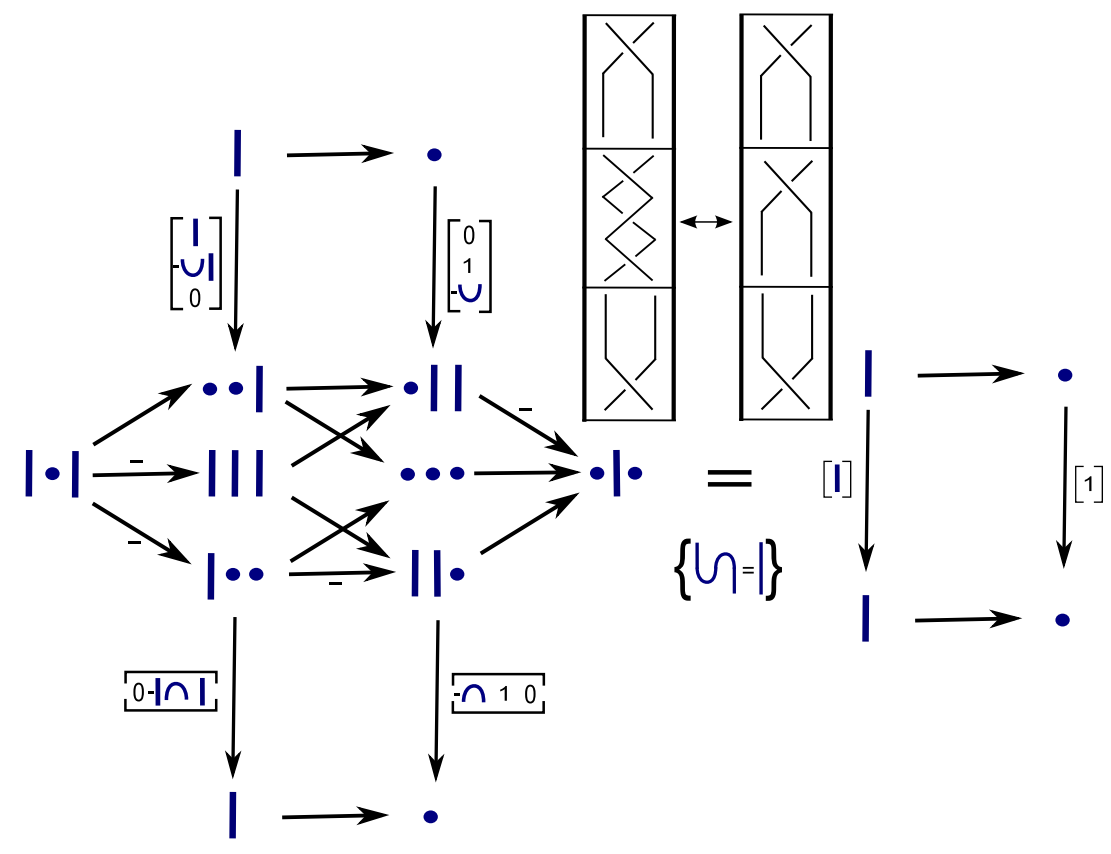

Figure 8: Movie Move 2

MM3. All 4 variants are essentially immediate after glancing at the slide generators, but we list one for posterity. 

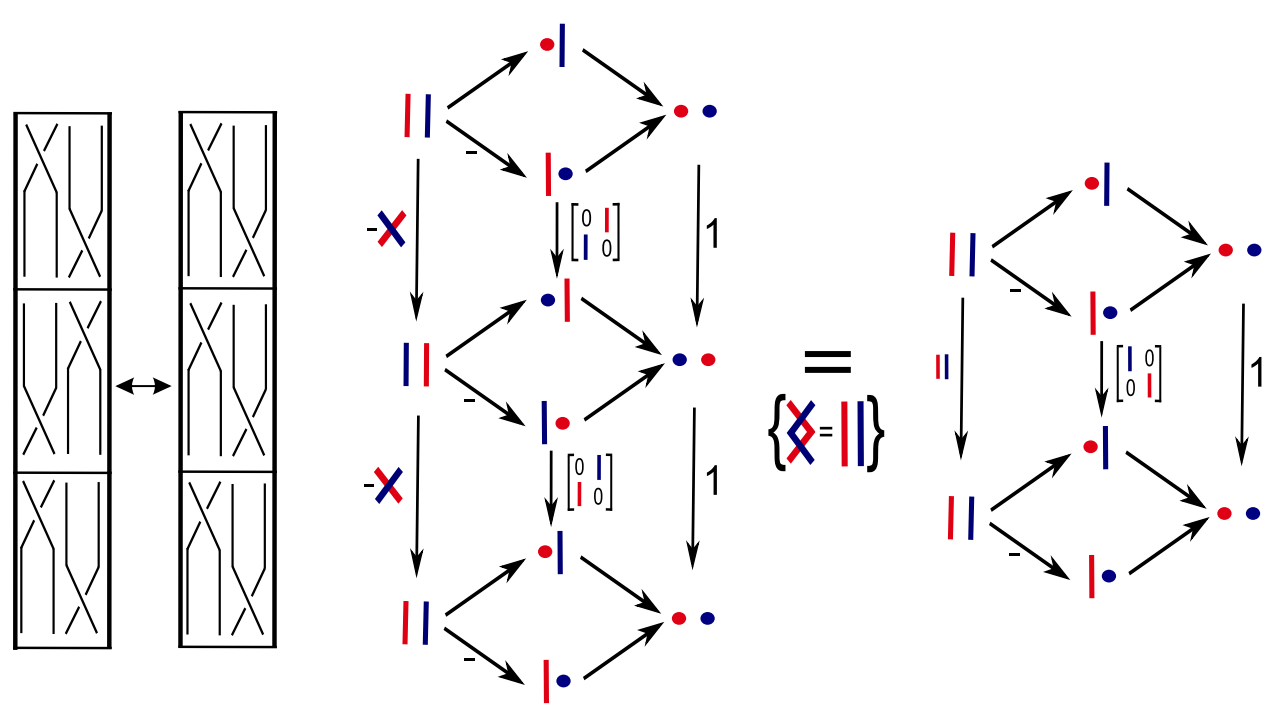

Figure 9: Movie Move 3

MM4. At this point the conscientious reader will find all 8 variants of movie move 4 quite easy, for the regularity of the slide chain maps allows one to write the compositions for the left and right-hand side at once. The maps only differ at the triple-color crossings, so we have to make use of relation (22).

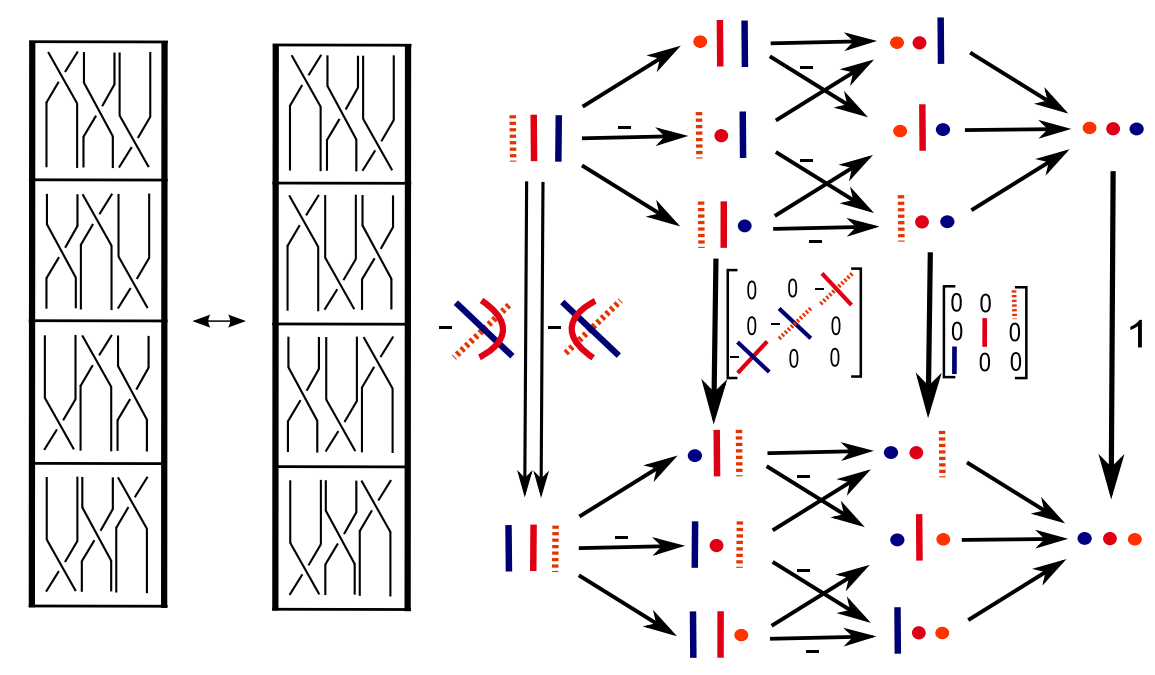

Figure 10: Movie Move 4

MM5. There are two variants of this movie, with the calculation for both almost identical. We consider one variant. The compostion has the following form: 

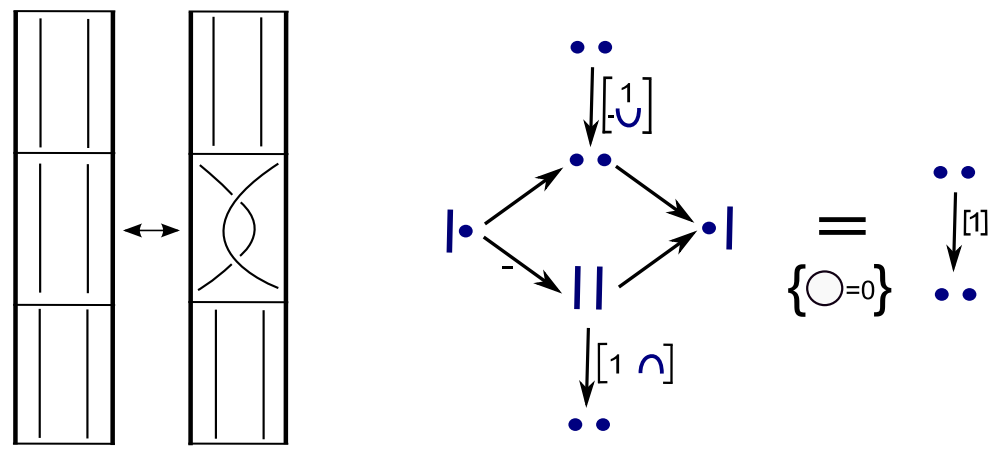

Figure 11: Movie Move 5

MM6. Again there are two variants and the calculation is almost as easy as the one for MM5; the only difference is that here we actually have to produce a homotopy. We check one variant; left arrows are the identity, right the composition, and dashed the homotopy. Checking that the homotopy works requires playing with relation (16).
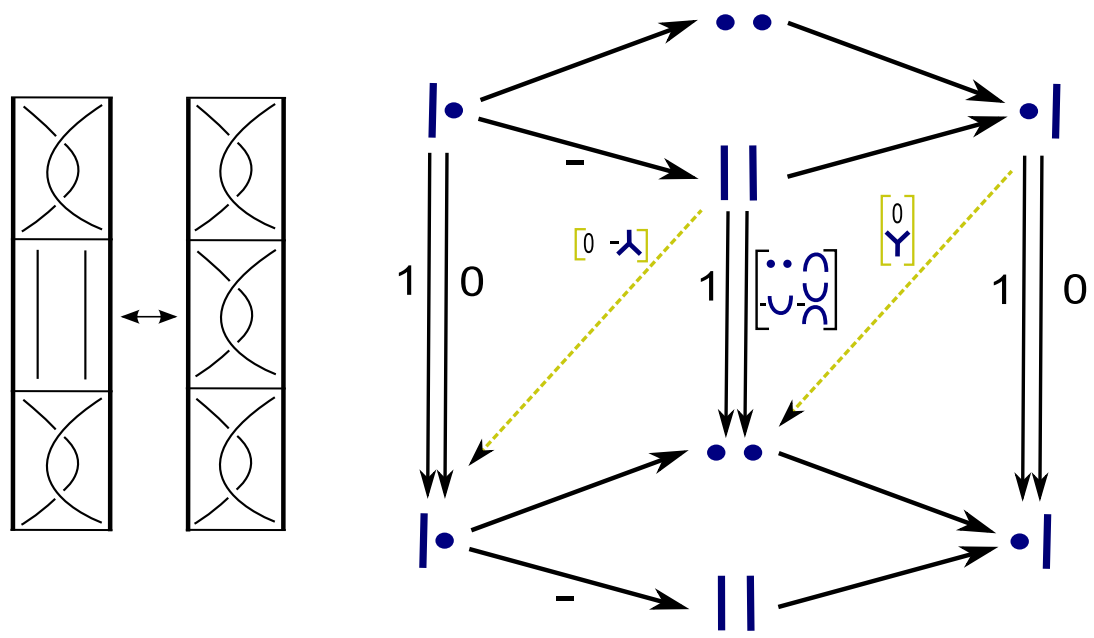

Figure 12: Movie Move 6

MM7. There are 6 variants of MM7, one for each R3 generator; nevertheless, this is still a bit of a drudge as each one requires a homotopy and a minor exercise in the relations. We display the movie associated to generator 1a and leave it to the very determined reader to repeat a very similar computation the remaining 5 times. The chain maps for the left-hand side of the movie are pictured in figure 13 at the top of the next page.

The composition and homotopy is in figure 14 at the top of page 137 . 


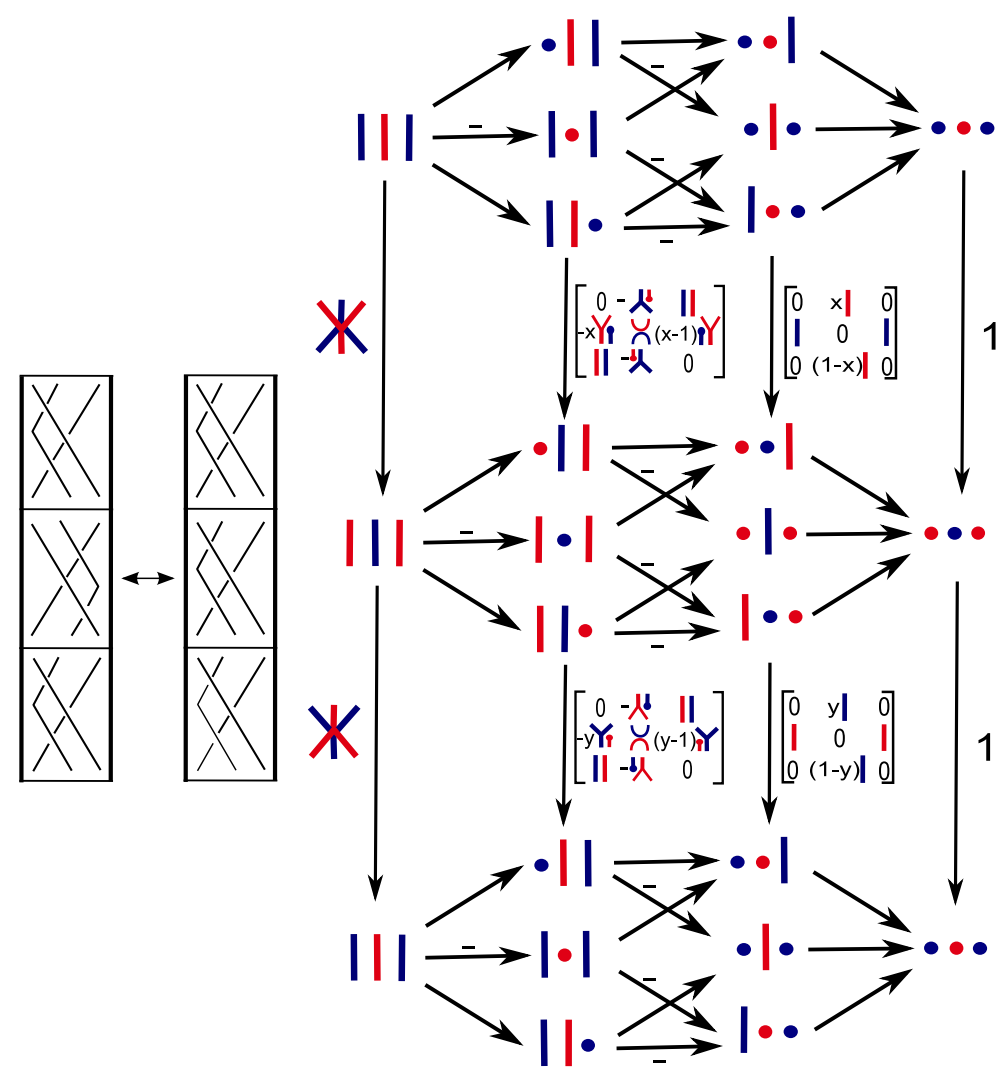

Figure 13: Movie Move 7

We now check that the prescribed maps give a homotopy between the composition and the identity. The verification for the left-most map is simply relation (24). The verification for the right-most map is immediate, and that of the third map is elementary. This leaves us with the second map. Here $d H+H d=$

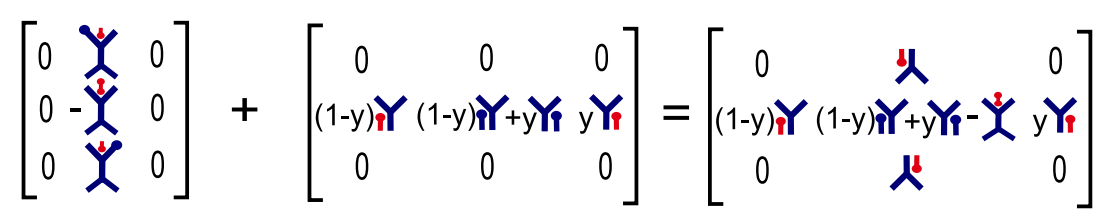

which save for the central entry is precisely the identity minus the composition. Equality of the central entry follows from the computation:

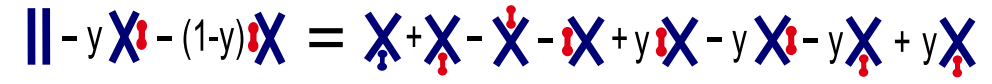

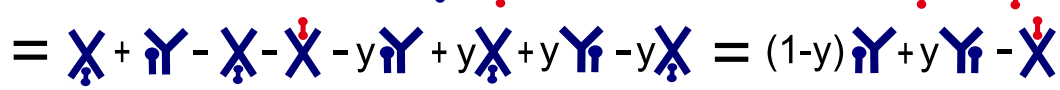

Note. This computation was done using relation (26) numerous times. 


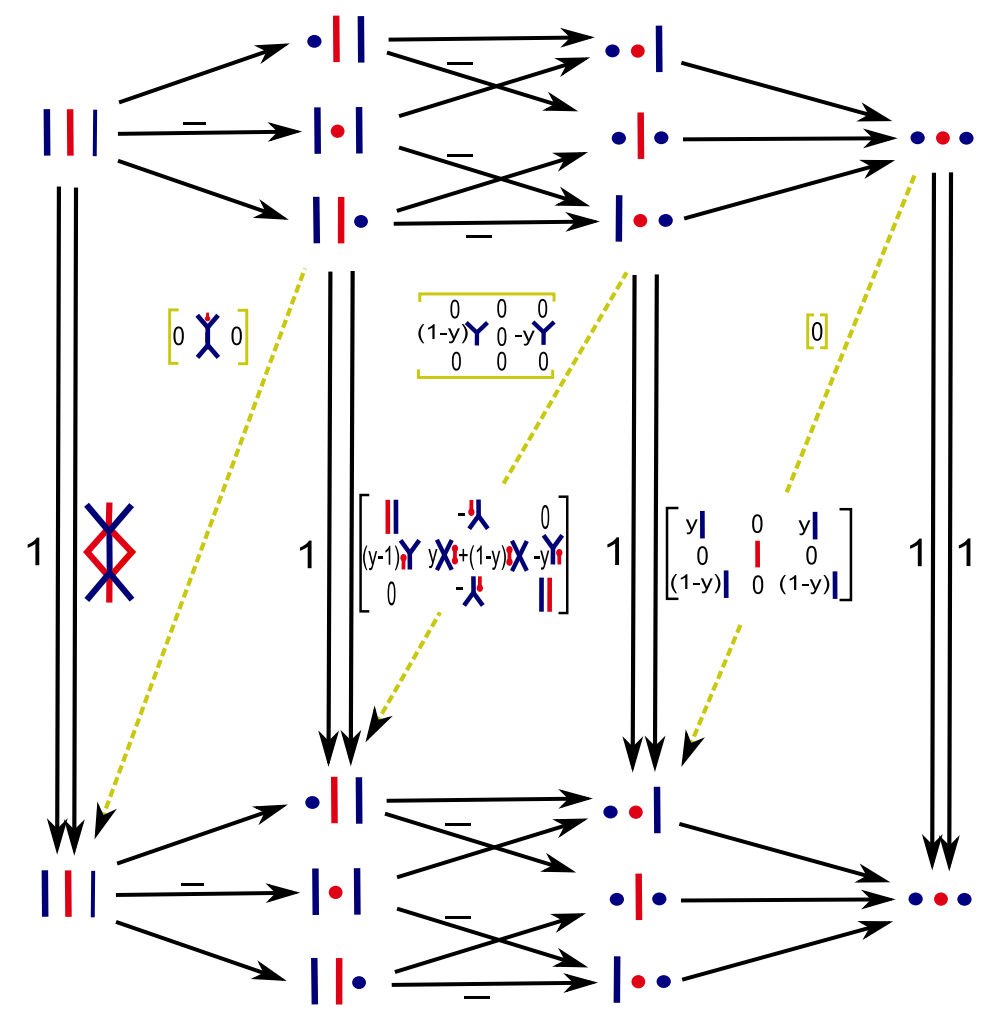

Figure 14: Homotopy for Movie Move 7

MM8. There are twelve variants of MM8, and in every case, one can find a homotopically isolated summand. As an example, we use the variant in figure 15 .

The different variants will change which summands are in which homological degree, but the total set of summands in each complex is unchanged. It is easy to check that the only possible degree -1 maps between any summand in the top complex and any summand in the bottom is a trivalent vertex from "blue" on top to "blue-blue" on bottom, or the same with red, or vice versa for the reverse movie. Moreover, only one of these appears in any given variant! This is because "blue-blue" and "red-red", viewed as bottom summands, require 4 switches to get from one to the other, meaning that they either occur in columns 0 and 4, in columns 1 and 3 , or both in column 2. Similarly, "blue" and "red", viewed as top summands, either occur in columns 0 and 2 , or both in column 1 . These choices are incompatible with the existence of both homotopies. This is also true of the reverse movie, which switches the role of top and bottom complexes.

In the forward movie where "red" and "blue" both appear in the middle column on top, the right column is homotopically isolated. In the forward movie where "red" appears on the left and "blue" on the right, one of these is homotopically isolated. In the reverse movie, $B_{\emptyset}$ is always homotopically isolated, since all homotopies kill it, and the differential will not bring it to "blue-blue" or "red-red". 


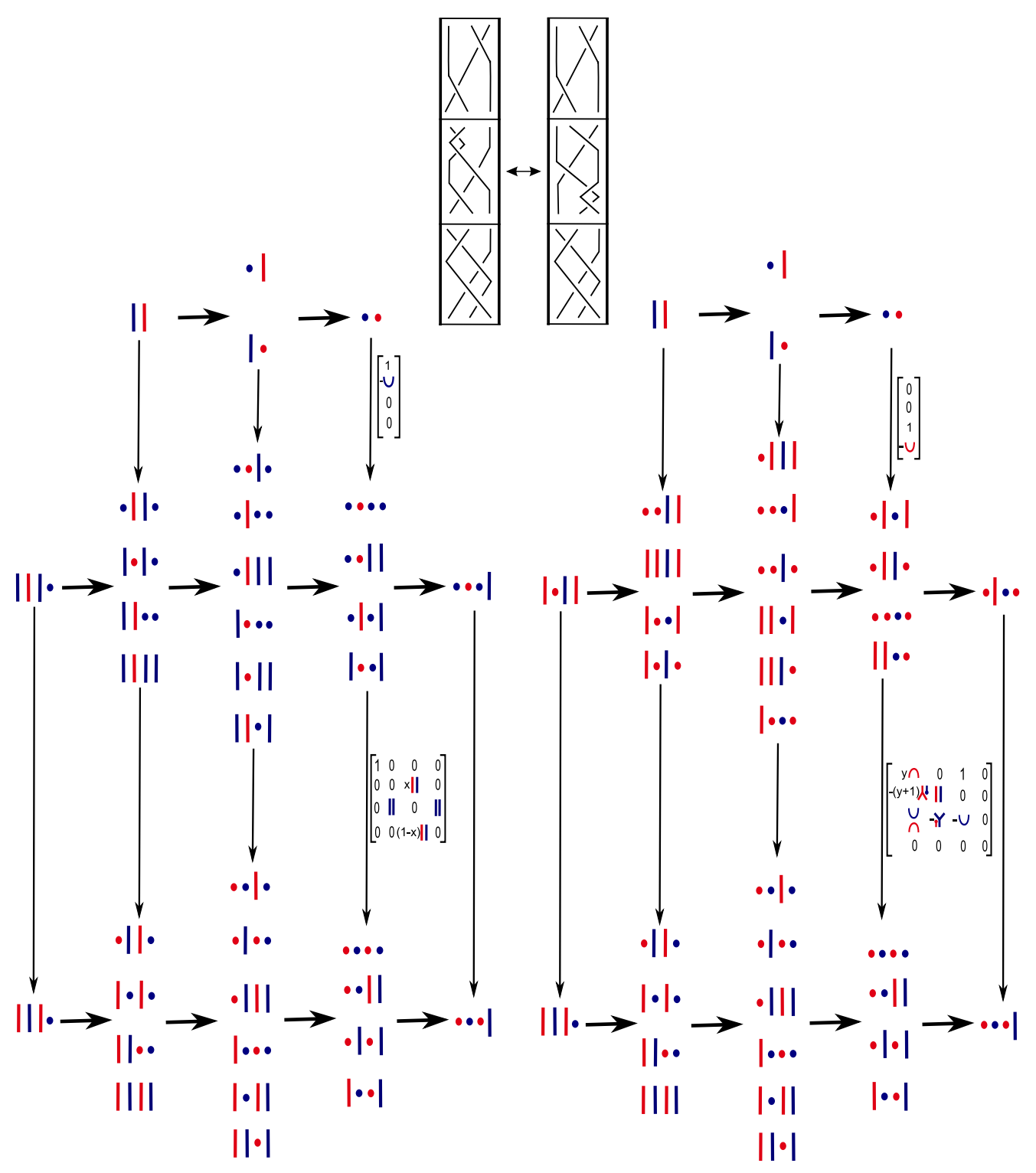

Figure 15: Movie Move 8 
Finally, one must calculate equality of the maps on that homotopically isolated summand. For the reverse movie, or any movie where $B_{\emptyset}$ is homotopically isolated, the equality of both sides is now clear. We give an example in Figure 15. For the remaining variants, this only requires multiplying two matrices, which we leave to the reader.

MM9. There are a frightful 48 versions of MM9, coming from all the different R3 moves that can be done (12 in all), the type of crossing that appears in the slide, and the vertical flips. Homotopically isolated summands come to the rescue. First, note that in every R3 generator, both the rightmost and the leftmost summands are homotopically isolated (and at least one of these is involved in a nonzero map). This is unchanged by the addition of a new distant color (call it orange) in MM9, so one can check equality on either the rightmost or the leftmost term, choosing one for which the chain maps are nonzero. If the distant color does not appear in this summand, then the chain map on that summand is precisely as in the R3 move, for both sides. If the distant color does appear, then a sign equal to the parity of the non-orange lines is introduced (which is equal on both sides), and we use the distant sliding rules to equate the corresponding morphisms.

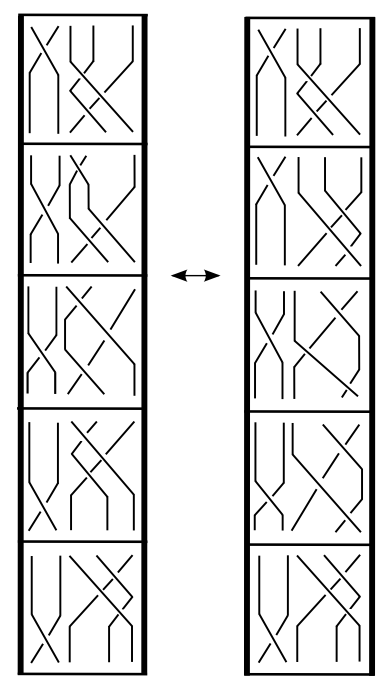

Figure 16: Movie Move 9

MM10. The sheer burden of writing down the complexes and calculating the chain maps for even one version of MM10 is best avoided at all costs. We have already checked MM10 in Remark 4.9.

MM11. There are 16 variants of MM11: 2 choices of crossing, a vertical flip, and the direction of the movie. Half of these have chain maps that compose to zero on both sides, since the birth of a right crossing or the death of a left crossing is the zero chain map. The rest are straighforward. We give an example below in figure 17 . 


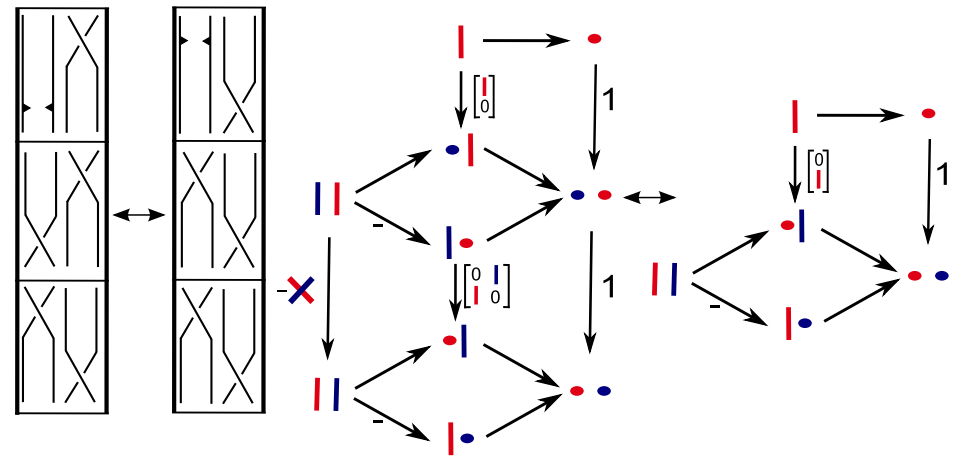

Figure 17: Movie Move 11

MM12. There are 8 variants: a choice of $\mathrm{R} 2$ move, a vertical flip, and the direction of the movie. Again, half of these are zero all around. Here are two variants; the other two are extremely similar.

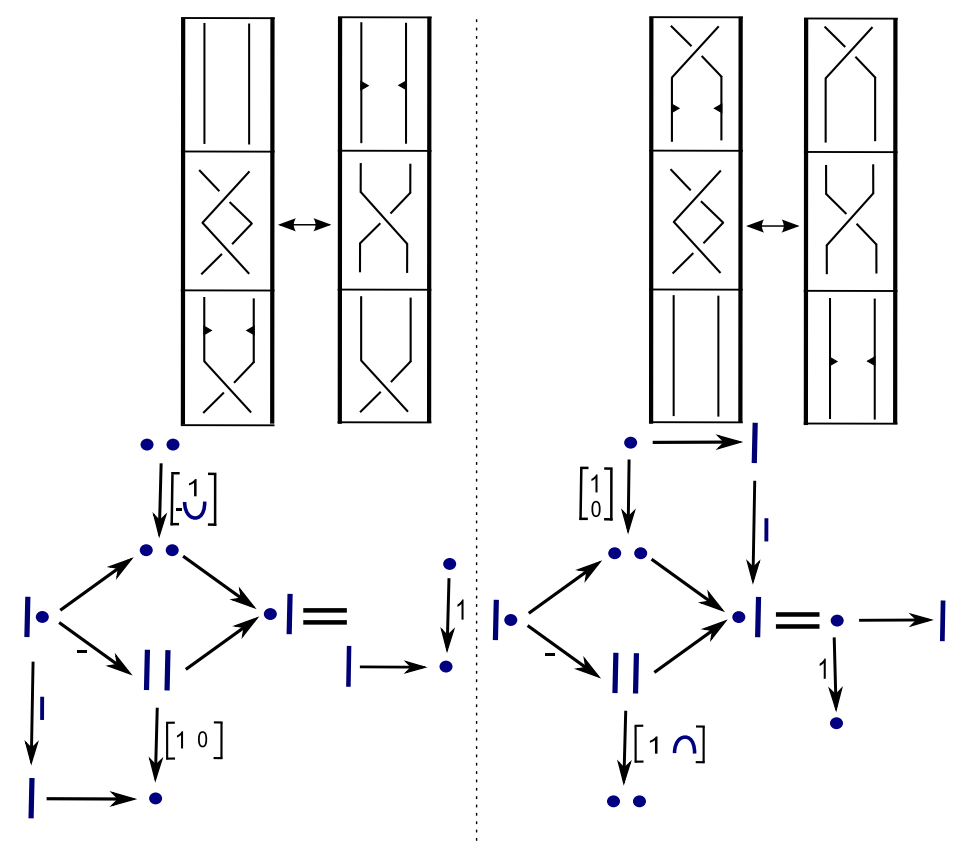

Figure 18: Movie Move 12

MM13. There are 24 variants, of which half are zero. For the remaining variants, the check requires little more than just writing down the composition, since the required homotopy in each instance is quite easy to guess. In figure 19 we describe the variant associated to the first R3 generator. 

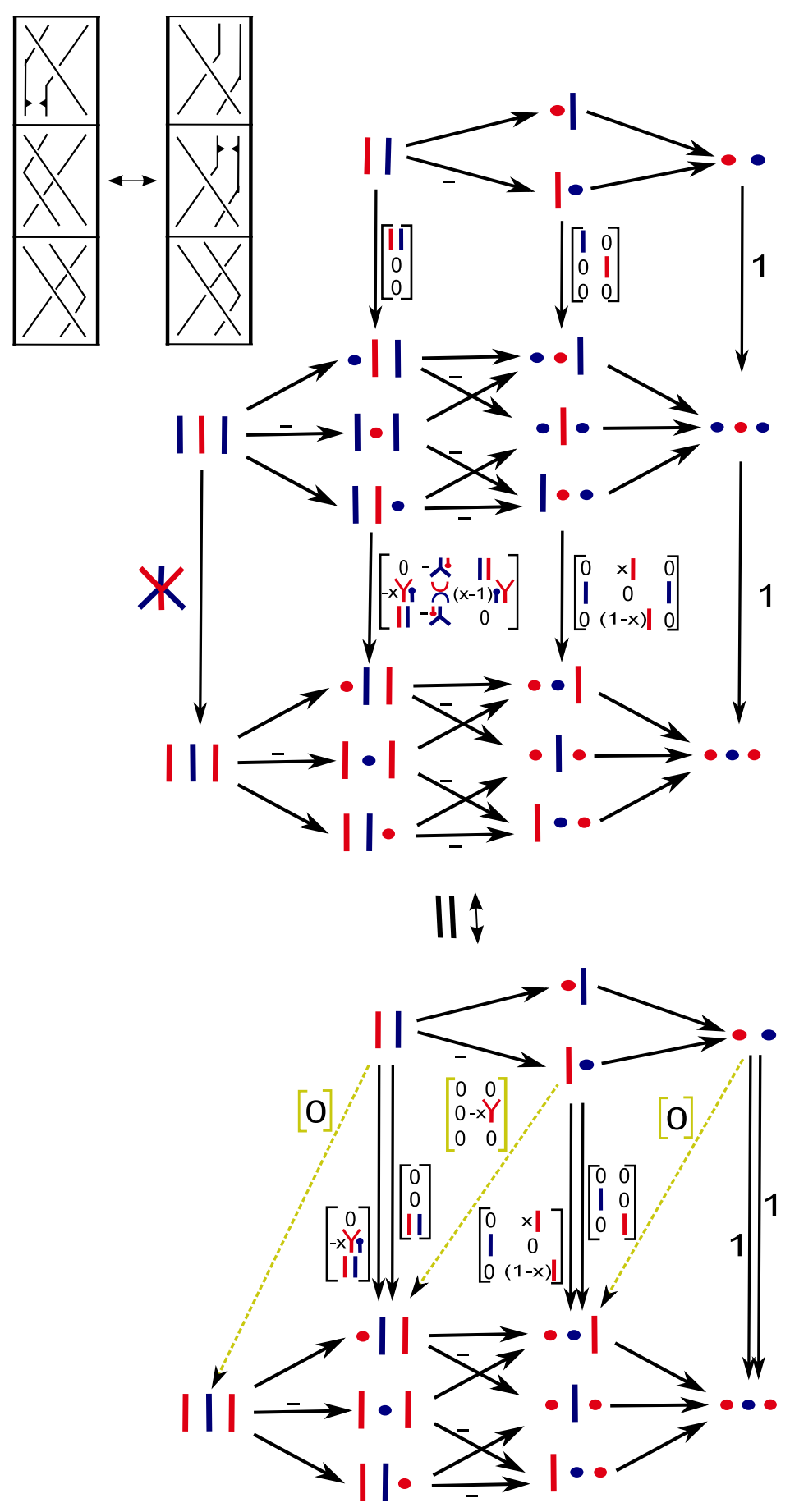

Figure 19: Movie Move 13 
MM14. Since none of the R3 generators of type 1 or 2 is compatible with MM14, we are left with 8 variants: 4 R3 generators and 2 directions (the vertical flip is irrelevant). Half are zero. In addition to this, the initial frame of the movie corresponds to a complex supported in homological degree 0 only, so we only need write down what happens there. In figure 20 below we describe the variant associated to the R3 generator $3 a$.

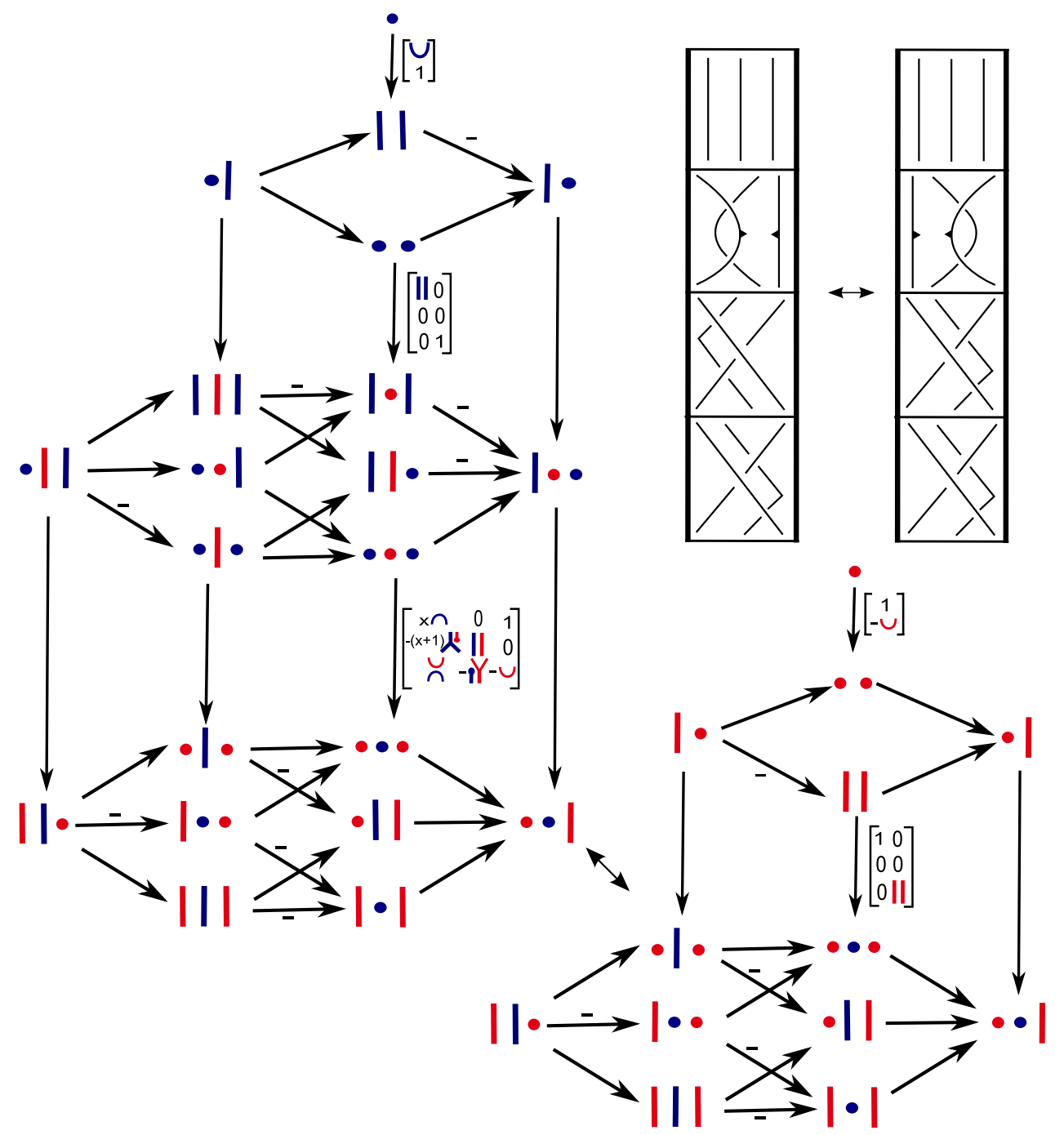

Figure 20: Movie Move 14

Any terms coming from the blue cup on the left will vanish due to (15). Therefore, both sides are equal to the last column of the last matrix on the left. 


\section{Additional Comments}

\subsection{The Benefits of Brute Force}

We have explicitly defined a functor from the braid cobordism category into the homotopy category of complexes in $\mathcal{S C}_{2}$. Even though our proof was not explicit, this implies that a brute force plug-and-chug proof would work. Thus we do not rely essentially on the fact that $\operatorname{Hom}\left(B_{\emptyset}, B_{\emptyset}\right)$ is 1-dimensional, even though the proofs do.

A consequence of this is that one can replace $\mathcal{S C}_{2}$ with any larger category $\mathcal{C}$ for which $\mathcal{S C}_{2}$ is a (non-full) subcategory, and we will still get a functor to the homotopy category of $\mathcal{C}$. There is no requirement that $\operatorname{Hom}\left(B_{\emptyset}, B_{\emptyset}\right)$ is 1-dimensional in $\mathcal{C}$. For instance, it would be interesting to define such a category $\mathcal{C}$ for which all birth and death maps are nontrivial (although the authors have yet to find an interesting extension of this type). Changing the birth and death maps would only require reproving movie moves 11 through 14 .

One other benefit to checking everything by hand is in knowing precisely which coefficients are required, and thus understanding the dependence on the base ring $\mathbb{k}$. In all the movie moves we check in this paper, and have checked personally, each differential, chain map, and homotopy has integral coefficients (or free variables which may be chosen to be integral). In fact, every nonzero coefficient that didn't involve a free variable was \pm 1 , and free variables may be chosen such that every coefficient is 1,0 , or -1 . The same should be true for MM8 through MM10 as well (Khovanov and Thomas [8] already showed that Rouquier complexes lift over $\mathbb{Z}$ to a projective functor, which implies the existence of homotopy maps over $\mathbb{Z}$ ). The next section discusses the definition of this functor in a $\mathbb{Z}$-linear category.

Checking the movie moves also provides some intuition as to why $\mathcal{S C}_{1}$ has the relations that it does. As an illustrative example, consider the overcrossing-only variation of Movie Move 10 and the unique summand of lowest (leftmost) homological degree: it is a sequence of 6 lines. On this summand, the left hand movie and the right hand movie correspond to the maps pictured in figure 21.

Thus equality of these two movies on the highest term, modulo relation (17), is exactly relation (27). Similarly, the highest terms in various other movie moves utilize the other relations, as in the chart below.

\begin{tabular}{|l|r|}
\hline MM & Relation \\
\hline 1 & $(11)$ \\
2 & $(8)$ \\
3 & $(17)$ \\
4 & $(22)$ \\
5 & $(15)$ \\
8 & $(12)$ \\
9 & $(21)$ \\
10 & $(27)$ \\
\hline
\end{tabular}

We can view these relations heuristically as planar holograms encoding the equality of cobordisms.

Type II movie moves (11 through 14) do not contribute any relations or requirements not already forced by Type I movie moves (although they do fix the sign of various generators as in Remark 3.4). 

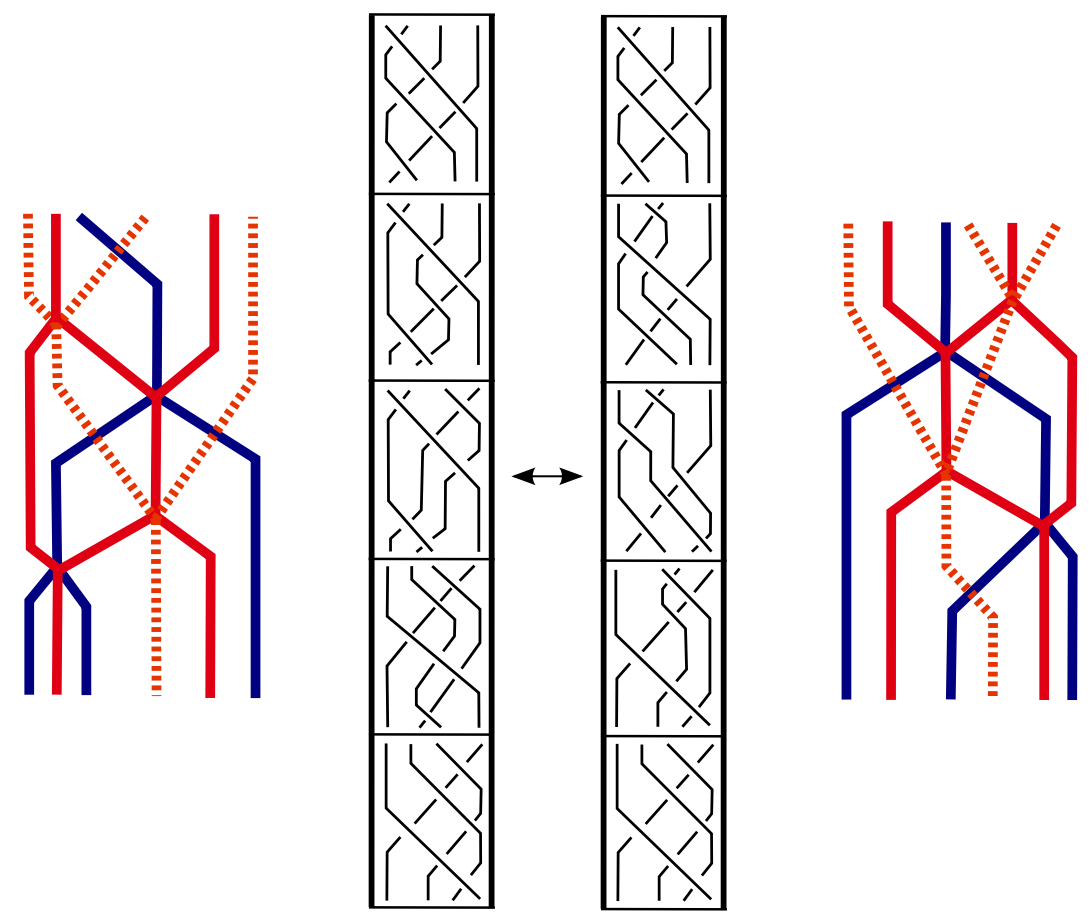

Figure 21: The most complicated term in MM10.

More relations are used to imply that certain maps are chain maps, or that homotopies work out correctly. For example, relation (18) is needed for the slide generator to be a chain map. Almost every relation in the calculus is used in a brute force check of functoriality. However, there are two exceptions: (13) and (25). Both these relations are in degree -2 , and degree -2 does not appear in chain maps or homotopies, so they could not have appeared. However, it is not hard to use the rest of the one color relations to show that

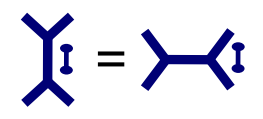

Hence, (13) will hold, so long as $R$ acts freely on morphisms. A similar statement can be made for $(25)$.

We do not present a proof, but the authors have convinced themselves of the following: Take an arbitrary categorification of the Hecke algebra with generators $B_{i}$, and construct Rouquier-style complexes. Under suitable assumptions on Hom spaces (we do not fix the pairing on $\mathcal{H}$, but assume $R$ acts freely, and the behavior of negative degree maps is the same), the existence of the above generators and the relations between them are implied by functoriality. We conjecture vaguely that $\mathcal{S C}_{1}$ is universal amongst categories for which Rouquier complexes could be defined functorially up to Type I movie moves, under suitable conditions. 


\subsection{Working over $\mathbb{Z}$}

Knot theorists should be interested in a $\mathbb{Z}$-linear version of the Soergel bimodule story, because it could theoretically yield a "functorial" link homology theory over $\mathbb{Z}$. Any thorough discussion would involve poring over the proofs in [4] for coefficients, so we provide no proofs.

Ignoring the second equality in (26), which is redundant, every relation given has coefficients in $\mathbb{Z}$. One could use these relations to define a $\mathbb{Z}$-linear version of $\mathcal{S C}_{1}$ and $\mathcal{S C}_{2}$, and then use base extension to define the category over any other ring. The functor can easily be defined over $\mathbb{Z}$, as we have demonstrated, and all the brute force checks work without resorting to other coefficients. Theorem 1.1 still holds for the $\mathbb{Z}$-linear version of $\mathcal{S C}_{2}$.

In fact, the same proof (using homotopically isolated summands) should work over $\mathbb{Z}$, so long as the arguments in $[4]$ which simplify graphs will also work over $\mathbb{Z}$. We would need to show that $\operatorname{Hom}\left(B_{\emptyset}, B_{\emptyset}\right)=\mathbb{Z}$ in the $\mathbb{Z}$-linear version of $\mathcal{S C}_{2}$, and that certain HOM spaces are zero in negative degrees. Graph simplification implies that the inclusion $\mathcal{S C}_{2}(I) \rightarrow \mathcal{S C}_{2}\left(I^{\prime}\right)$ is fully faithful after base change, so we may enlarge our index set $I$, and assume that $I=\{1, \ldots, n\}$. We can calculate Hom spaces from a pairing on $\mathcal{H}$, so long as Hom spaces are torsion-free and $\mathcal{S C}_{2}$ actually categorifies $\mathcal{H}$. One begins by checking the isomorphisms (4) through (6). The only one which is in doubt is $B_{i} \otimes B_{i} \cong B_{i}\{-1\} \oplus B_{i}\{1\}$, since (28) requires the use of $\frac{1}{2}$ in order to split the identity map into idempotents. Thankfully, we may use (29) as a replacement (and our enlarged index set guarantees that some color adjacent to $i$ exists).

Remark 5.1. This statement does not imply that $\mathcal{S C}$ will categorify the Hecke algebra when defined over $\mathbb{Z}$. There may be missing idempotents, or extra non-isomorphic idempotents, so that the Grothendieck ring of the idempotent completion may be too big or small.

\section{References}

[1] D. Bar-Natan and S. Morrison, The Karoubi envelope and Lee's degeneration of Khovanov homology, Algebr. Geom. Topol. 6 (2006), 1459-1469.

[2] J. Carter and M. Saito, Knotted surfaces and their diagrams, Math. Surv. and Mon. 55, Amer. Math. Soc., Providence, RI, 1998.

[3] D. Clark, S. Morrison and K. Walker, Fixing the functoriality of Khovanov homology, Geom. Topol. 13 (2009), no. 3, 1499-1582.

[4] B. Elias and M. Khovanov, Diagrammatics for Soergel categories, preprint 2009, arXiv:0902.4700v1.

[5] M. Khovanov, Triply-graded link homology and Hochschild homology of Soergel bimodules, Internat. J. Math. 18 (2007), no. 8, 869-885.

[6] M. Khovanov and L. Rozansky, Matrix factorizations and link homology, Fund. Math. 199 (2008), no. 1, 1-91.

[7] M. Khovanov and L. Rozansky, Matrix factorizations and link homology II, Geom. Topol. 12 (2008), no. 3, 1387-1425.

[8] M. Khovanov and R. Thomas, Braid cobordisms, triangulated categories, and flag varieties, Homology, Homotopy Appl. 9 (2007), no. 2, 19-94. 
[9] D. Krasner, Integral HOMFLY-PT and $s l(n)$-link homology, preprint 2009, arXiv:0910.1790v1.

[10] M. Mackaay and P. Vaz, The reduced HOMFLY-PT homology for the Conway and the Kinoshita-Terasaka knots, preprint 2008, arXiv:0812.1957v1.

[11] J. Rasmussen, Khovanov-Rozansky homology of two-bridge knots and links, Duke Math. J. 136 (2007), no. 3, 551-583.

[12] J. Rasmussen, Some differentials on Khovanov-Rozansky homology, preprint 2006, arXiv:math/0607544v2.

[13] R. Rouquier, Categorification of the braid groups, preprint 2004, arXiv:math/0409593.

[14] W. Soergel, The combinatorics of Harish-Chandra bimodules, Journal Reine Angew. Math. 429, (1992) 49-74.

[15] W. Soergel, Gradings on representation categories, roceedings of the International Congress of Mathematicians, Vol. 1, 2 (Zürich, 1994), 800-806, Birkhäuser, Basel, 1995.

[16] W. Soergel, Combinatorics of Harish-Chandra modules, Representation theories and algebraic geometry (Montreal, PQ, 1997), 401-412, NATO Adv. Sci. Inst. Ser. C Math. Phys. Sci., 514, Kluwer Acad. Publ., Dordrecht, 1998.

[17] W. Soergel, Kazhdan-Lusztig-Polynome und unzerlegbare Bimoduln über Polynomringen, preprint 2004, arXiv:math/0403496v2. English translation available on the author's webpage.

[18] B. Webster, Khovanov-Rozansky homology via a canopolis formalism, Algebr. Geom. Topol. 7 (2007), 673-699.

[19] B. Webster, Kr.m2, http://katlas.math.toronto.edu/wiki/user:Ben/ KRhomology, 2005.

Ben Elias belias@math.columbia.edu

Mathematics Department, Columbia University, Room 509 MC4406, 2990 Broadway, New York, NY 10027, USA

Dan Krasner dkrasner@math.columbia.edu

Mathematics Department, Columbia University, Room 509 MC4406, 2990 Broadway, New York, NY 10027, USA 